\title{
Multifocus image fusion based on waveatom transform
}

\author{
MEENU MANCHANDA ${ }^{1}$ and DEEPAK GAMBHIR ${ }^{2, *}{ }^{*}$ \\ ${ }^{1}$ Department of Electronics and Communications Engineering, Vaish College of Engineering, Rohtak 124001, \\ India \\ ${ }^{2}$ Department of Electronics and Communications Engineering, Amity School of Engineering and Technology, \\ Bijwasan, New Delhi, India \\ e-mail: meenumanchanda73@gmail.com
}

MS received 24 November 2017; revised 15 August 2018; accepted 19 August 2018; published online 1 February 2019

\begin{abstract}
Multifocus image fusion has emerged as a challenging research area due to the availability of various image-capturing devices. The optical lenses that are widely utilized in image-capturing devices have limited 'depth-of-focus' and, therefore, only the objects that lie within a particular depth remain 'in-focus', whereas all the other objects go 'out-of-focus'. In order to obtain an image where all the objects are well focused, multifocus image fusion method based on waveatom transform is proposed. The core idea is to decompose all input images using waveatom transform and perform fusion of resultant waveatom coefficients. The waveatom coefficients with higher visibility, corresponding to sharper image intensities, are used to perform the process of image fusion. Finally, the fused image is obtained by performing inverse waveatom transform. The performance of the proposed method is demonstrated by performing fusion on different sets of multifocus images and comparing the results of the proposed method to the results of existing image fusion methods.
\end{abstract}

Keywords. Waveatom transform; multifocus image fusion; quality measures.

\section{Introduction}

Various image-capturing devices are used to capture different images of the same scene or of an object. In order to capture image of a complex scene, it is desired that all the objects of the scene remain 'in-focus'. However, due to limited depth-of-focus of optical lenses used in imaging devices, only the objects that lie within a particular depth remain 'in-focus', whereas all the other objects go 'out-offocus'. Therefore, multiple partially focused images are captured and these images are fused together to bring all the objects 'well-in-focus' in a single image [1,2]. This 'all-infocus' image can be obtained through the process of image fusion. Image fusion can be defined as the process of combining relevant information from multiple input images into a single fused image that is more informative in terms of visual perception. The process of image fusion should preserve all relevant information in the fused image and should simultaneously suppress irrelevant information contained in input images [3].

A variety of multifocus image fusion methods have been proposed. These methods can broadly be classified into [4] pixel-based method, feature-based method and decisionbased method. The first class of image fusion method combines the raw input images according to some

*For correspondence predefined fusion criteria, to yield a highly informative fused image. The second class of image fusion method combines the input images according to some salient features such as edges or regions of similar texture or intensity. The third class of image fusion method produces a fused image by combining visual descriptions directly in the form of probabilistic variables or relational graphs. However, the latter two classes of fusion methods are very much image and application dependent and therefore, the first class of image fusion method, i.e., pixel-based fusion method [5, 6], has attracted a great deal of researchers' attention. Pixelbased fusion can be performed either in spatial-domain or in transform-domain. In spatial-domain, fusion is done directly on image pixels or on image regions. Image fusion methods based on averaging, principal components and high pass filtering [7] are most commonly used spatialdomain image fusion methods. The significant advantages of spatial-domain image fusion methods are fast implementation and low computational complexity. However, spatial-domain image fusion methods are often subject to poor SNR, reduced contrast and blocking artefacts. In transform-domain image fusion methods, input images are first mapped into transform-domain and then fusion takes place. The fused image is finally obtained using inversetransformation of the fusion result. Among the various transform-domain fusion methods, pyramid-transformbased image fusion methods [8] and wavelet-transform- 
based image fusion methods [9, 10] are the most popular methods. However, pyramid transforms fail in providing spatial orientation selectivity during the decomposition process, thereby resulting in blocking artefacts in the reconstructed image. Further, wavelet transforms are good at isolated discontinuities but fail at edges and textured regions. Moreover, wavelet transforms have limited directional selectivity (either in three directions or in six directions). To overcome the missing directional selectivity of wavelet transforms, several multiscale geometric analysis transforms were developed as seen in the literature. In this series, Candes and Donoho developed curvelet transform [11] that represents curve as a combination of bases of different lengths and widths obeying the scaling law. The curvelet transform is however successful in representing curve-like edges but is not optimal for sparse approximation of curve features beyond $C^{2}$-singularities and also cannot be designed directly into digital domain. Further in this series, contourlet transform was proposed by Do and Vetterli to deal effectively with images where sparse expansion is expressed by contour segments. However, due to downsampling operation, the contourlet transform lacks shift invariance and causes pseudo-Gibbs phenomenon in the reconstructed image. Cunha et al proposed the nonsubsampled contourlet transform (NSCT) [12] that inherits the main benefits of contourlet transform, while simultaneously possessing the property of shift invariance as well as effectively suppressing the Pseudo-Gibbs phenomena. However, NSCT has limited number of directions and is highly computationally complex. To provide an efficient representation of images with edges, shearlet transform and its shift-invariant version [13] were also introduced in the literature of multiscale geometric analysis transforms.

Another efficient multiscale geometric transform based on waveatoms was introduced by Demanet and Ying [14]. Waveatom transform has significantly sparser expansion for oscillatory functions in comparison with wavelet transform and curvelet transform. Since the potential capability of waveatoms has been explored in many applications such as image denoising, fingerprint identification, compression, watermarking, etc. [15-18], waveatom-transform-based multifocus image fusion is proposed in the paper.

\section{Waveatom transform}

Waveatom transform is a recent development in multiscale geometric transformation system. It has sharp frequency localization and provides significant sparse representation for oscillatory patterns in image processing. Waveatoms interpolate exactly between Gabor atoms (diameter $\propto$ constant) and directional wavelets (diameter $\propto$ wavelength) in a sense that the wavelength (i.e., the period of oscillations) of each wave packet and the size of the essential support (i.e. the diameter) obey parabolic scaling relation, i.e., diameter $\propto$ wavelength ${ }^{2}$. Waveatom transform performs better than other wave packets such as wavelets, Gabor atoms and curvelet transforms as it makes tradeoff between two competing features of wave packets: warping invariance (i.e., the ability to adapt to arbitrary local directions of a pattern) and directionality (i.e., the ability to sparsely represent anisotropic patterns aligned with the axis). Two parameters are sufficient to differentiate among various forms of wave packets: the parameter ' $M s$ ' that indicates whether the decomposition is multiscale (for $M s=1$ ) or not (for $M s=0$ ) and the parameter ' $D r$ ' that indicates whether the basis elements are localized and poorly directional (for $D r=1$ ) or extended and fully directional $(D r=0)$. Figure 1 shows classification of various transforms as $(M s, D r)$ forms of wave packets. Sparsity can be preserved under warpings provided that the frame of wave packets satisfies $\frac{1}{2} \geq D r \geq M s$ ) whereas for sparse representation of oscillatory patterns, Gabor atoms $(M s=D r=0)$ is an ideal choice. Both of these requirements are contradictory and therefore a good compromise is achieved with $M s=\frac{1}{2}$ and $D r=\frac{1}{2}$, which corresponds to waveatoms.

Implementation of waveatoms: Let $f(x)$ and $\hat{f}(\omega)$ form a Fourier transform pair where $x$ and $\omega$ correspond to the coordinates in time and frequency domains, respectively.

In practice, one-dimensional (1D) waveatoms are obtained from the tensor products of special kind of 1D wave packets, $\varphi_{m, n}^{k}(x)$, where $k$ represents the scale of resolution, $m$ represents location in frequency and $n$ represents location in time. All the three quantities $k, m$ and $n$ are integer valued and index a point $\left(x_{k, n}, \omega_{k, m}\right)$ in phase-space, as $x_{k, n}=2^{-k} n$ and $\pm \omega_{k, m}= \pm \pi 2^{k} m$.

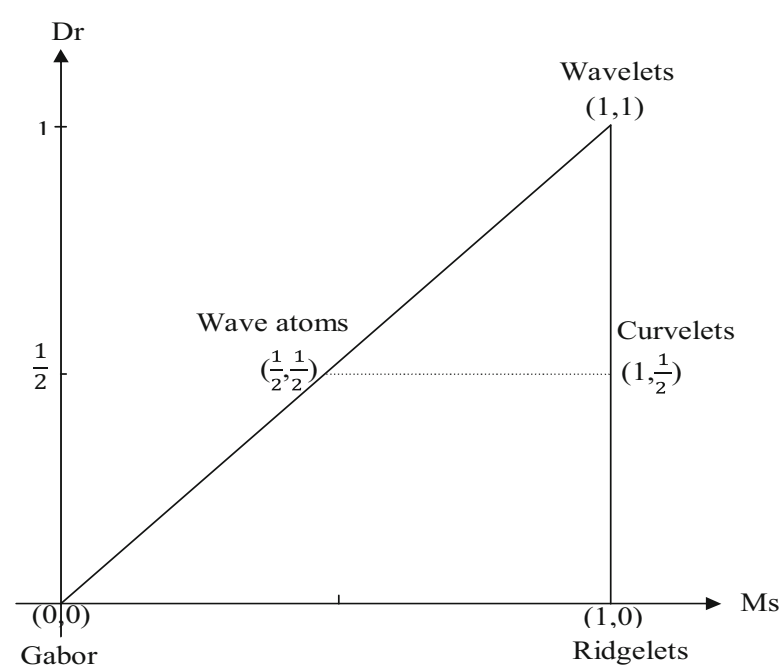

Figure 1. Classification of various transforms in $(M s, D r)$ plane. 


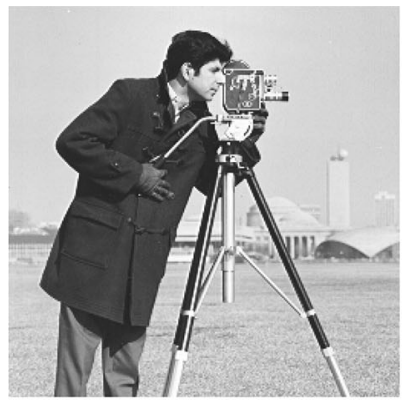

(a)

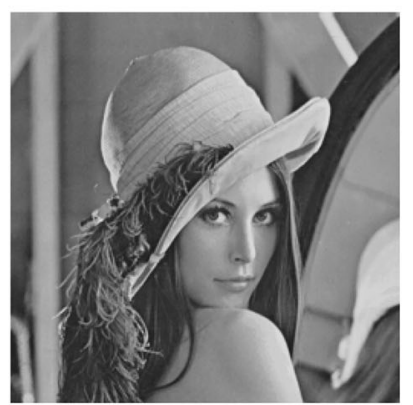

(e)

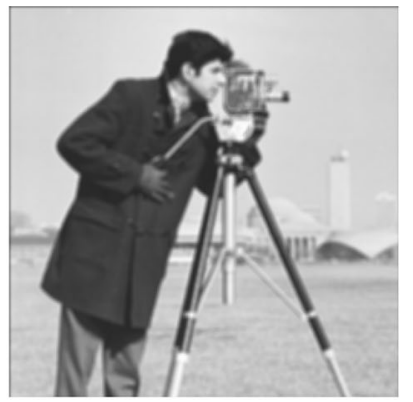

(b)

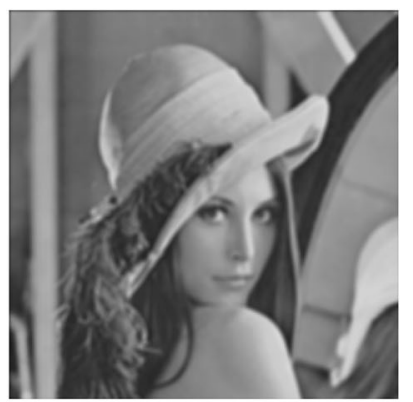

(f)

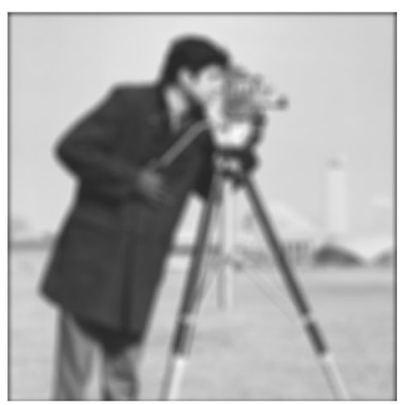

(c)

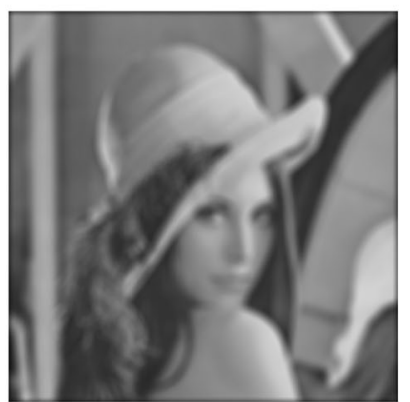

(g)

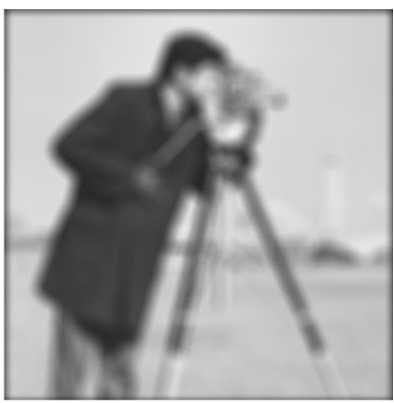

(d)

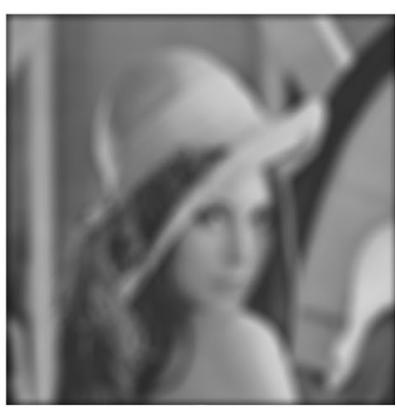

(h)

Figure 2. Degradation of images by circular averaging filter with different radii: (a) Cameraman (original, i.e., radius $=0$ ), (b) degraded Cameraman (radius $=2)$, $(\mathbf{c})$ degraded Cameraman (radius $=4),(\mathbf{d})$ degraded Cameraman (radius $=6)$, $(\mathbf{e})$ Lena $($ original, i.e., radius $=$ 0), (f) degraded Lena (radius $=2$ ), (g) degraded Lena (radius $=4$ ) and $(\mathbf{h})$ degraded Lena (radius $=6$ ).

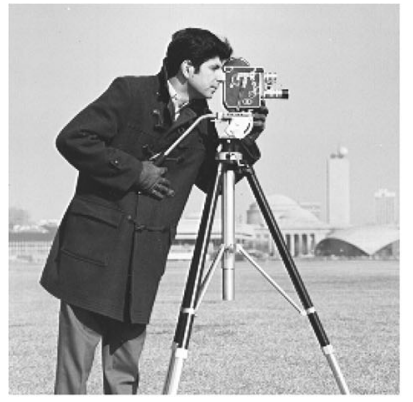

(a)

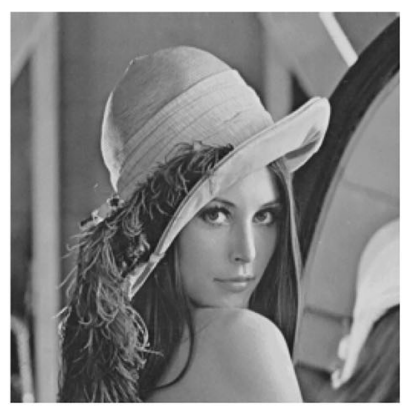

(e)

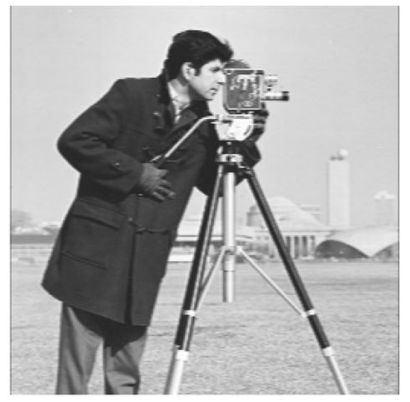

(b)

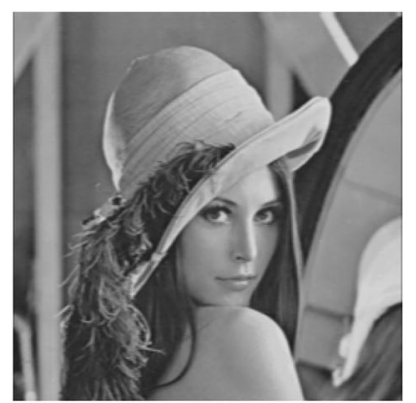

(f)

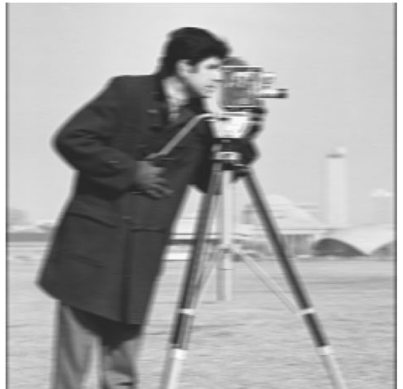

(c)

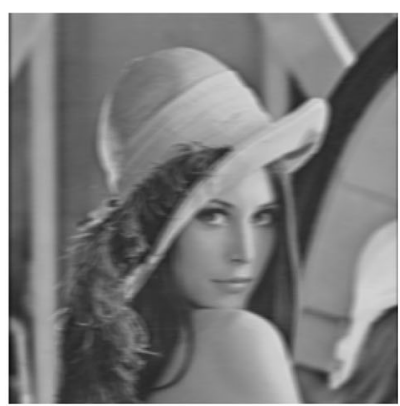

(g)

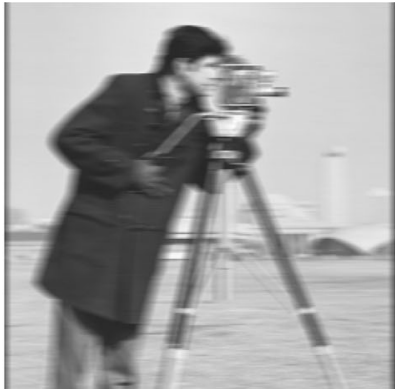

(d)

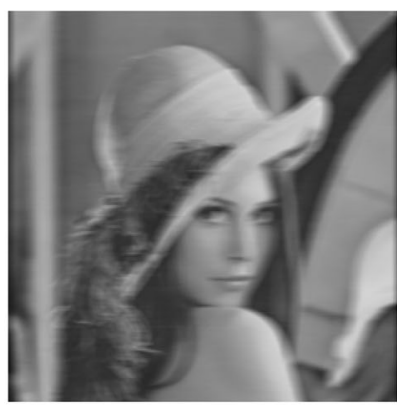

(h)

Figure 3. Degradation of images by motion blur with psf of different lengths: (a) Cameraman (original, i.e., length $=0)$, $(\mathbf{b})$ degraded Cameraman (length $=2)$, $(\mathbf{c})$ degraded Cameraman (length $=6),(\mathbf{d})$ degraded (length $=10)$, $(\mathbf{e})$ Lena (original, i.e., length $=0)$, (f) degraded Lena (length $=2)$, $(\mathbf{g})$ degraded Lena $($ length $=6)$ and $(\mathbf{h})$ degraded Lena $($ length $=10)$. 


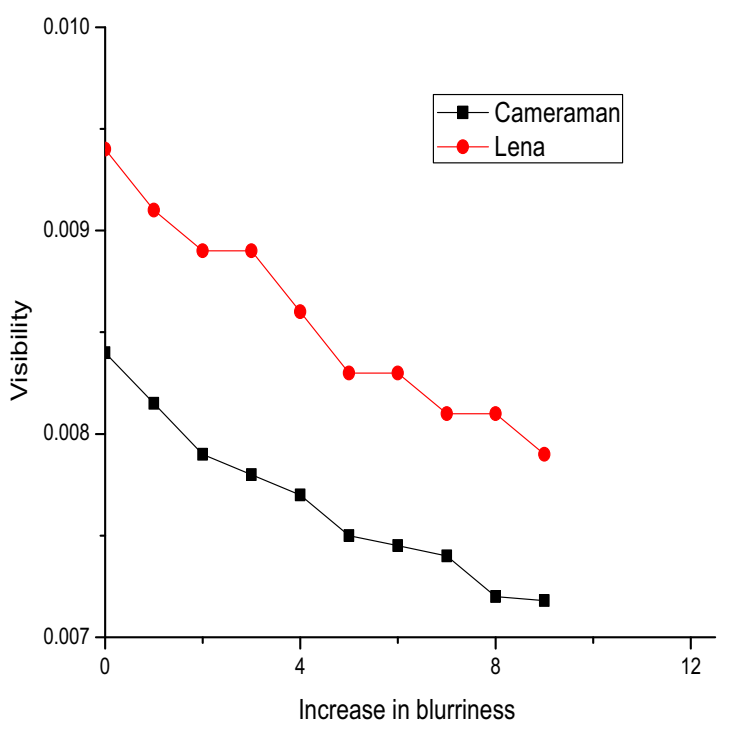

(a)

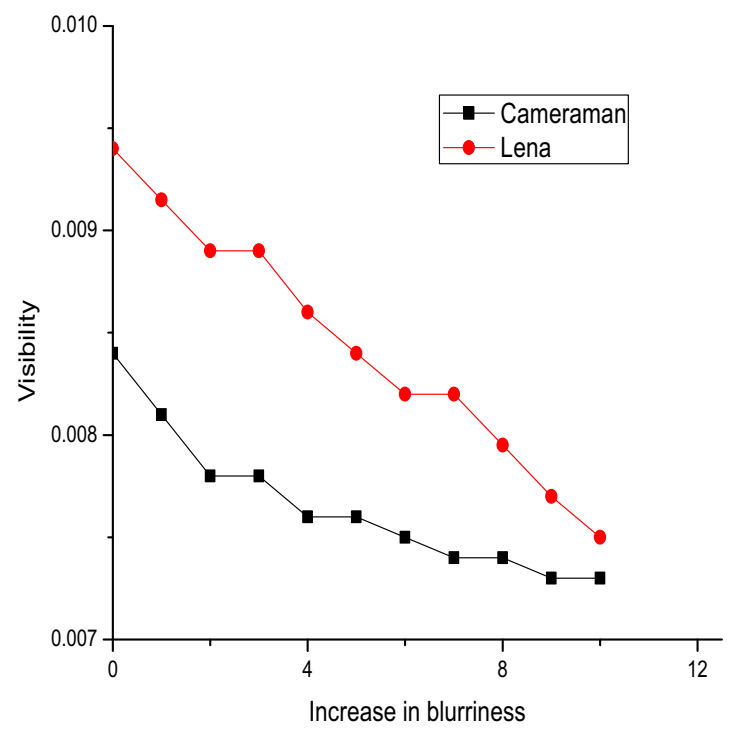

(b)

Figure 4. Variation of visibility with respect to blurriness ( 0 denotes the original or un-degraded image) due to (a) change in radius (in case of circular averaging filter) and (b) change in length (in case of motion blur).

The basis function can be obtained by combining dyadic scaled and translated versions of symmetric pairs of compactly supported bumps in frequency, $\hat{\varphi}_{m}^{0}(\omega)$ as follows:

$$
\varphi_{m, n}^{k}(x)=\varphi_{m}^{k}\left(x-2^{k} n\right)=2^{\frac{k}{2}} \varphi_{m}^{0}\left(2^{k} x-n\right)
$$

where

$$
\begin{aligned}
\hat{\varphi}_{m}^{0}(\omega)= & e^{-j \omega / 2}\left[e^{j a_{m}} f\left(b_{m}\left(\omega-2 a_{m}\right)\right)\right. \\
& \left.+e^{-j a_{m}} f\left(b_{m+1}\left(\omega+2 a_{m}\right)\right)\right]
\end{aligned}
$$

where $a_{m}=\left(m+\frac{1}{2}\right) \frac{\pi}{2}$ and $b_{m}=(-1)^{m}$. The function $f$ is a real-valued bump function compactly supported on a $2 \pi$ interval and selected such that

$$
\sum_{m}\left|\hat{\varphi}_{m}^{0}(\omega)\right|^{2}=1
$$

Two-dimensional (2D) waveatoms can be obtained by individually taking tensor products of $1 \mathrm{D}$ waveatoms.

1. An orthonormal basis: 2D orthonormal basis function can be obtained by individually taking tensor products of 1D waveatoms, i.e.

$$
\varphi_{\mu}^{k+}\left(x_{1}, x_{2}\right)=\varphi_{m_{1}}^{k}\left(x_{1}-2^{-k} n_{1}\right) \varphi_{m_{2}}^{k}\left(x_{2}-2^{-k} n_{2}\right)
$$

where $\mu=\left(m_{1}, m_{2}, n_{1}, n_{2}\right)$. Its Fourier transform is also separable and is given by

$$
\hat{\varphi}_{\mu}^{k+}\left(\omega_{1}, \omega_{2}\right)=\hat{\varphi}_{m_{1}}^{k}\left(\omega_{1}\right) e^{-j 2^{k} n_{1} x_{1}} \hat{\varphi}_{m_{2}}^{k}\left(\omega_{2}\right) e^{-j 2^{k} n_{2} x_{2}}
$$

where $\hat{\varphi}_{m_{1}}^{k}\left(\omega_{1}\right)$ and $\hat{\varphi}_{m_{2}}^{k}\left(\omega_{2}\right)$ are the Fourier transforms of $\varphi_{m_{1}}^{k}\left(x_{1}\right)$ and $\varphi_{m_{1}}^{k}\left(x_{2}\right)$, respectively. Thus, a $2 \mathrm{D}$ orthonormal basis function oscillates in two mutually

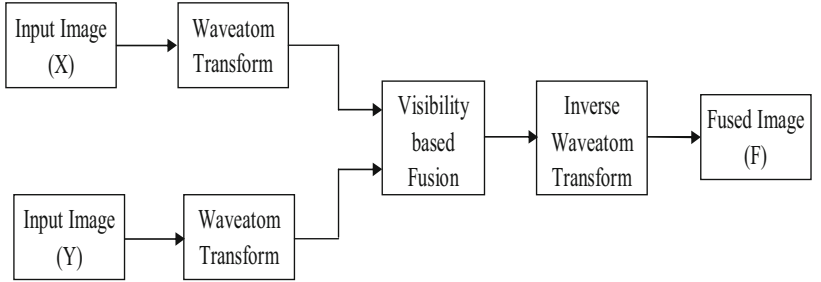

Figure 5. Block diagram of proposed algorithm.

perpendicular directions, thereby producing four lobes in the frequency domain.

A dual orthonormal basis function can be defined using Hilbert transform:

$$
\varphi_{\mu}^{k-}\left(x_{1}, x_{2}\right)=H\left[\varphi_{m_{1}}^{k}\left(x_{1}-2^{-k} n_{1}\right)\right] H\left[\varphi_{m_{2}}^{k}\left(x_{2}-2^{-k} n_{2}\right)\right]
$$

where $H[f(x)]$ represents the Hilbert transform of a function $f(x)$.

2. Directional waveatoms: The main disadvantage of orthonormal basis functions is that they oscillate in two different directions instead of oscillating only in one direction, i.e., $x$ direction. This problem can be solved by combining the primal (Eq. (3)) and its dual, i.e., Hilbert transformed basis (Eq. (5)). This combination

$$
\varphi_{\mu}^{(1)}=\frac{\varphi_{\mu}^{+}+\varphi_{\mu}^{-}}{2} \quad \text { and } \quad \varphi_{\mu}^{(2)}=\frac{\varphi_{\mu}^{+}-\varphi_{\mu}^{-}}{2}
$$

forms a tight frame and provides basis functions with two lobes symmetric with respect to origin in the 
frequency domain to form directional waveatoms. This type of waveatom oscillates in one direction instead of two and this directionality is achieved at the cost of increased redundancy by a factor of two.

3. The complex waveatom: The complex waveatom is constructed by isolating the symmetric lobes in the frequency domain. The breaking of symmetry in the frequency domain results in exact shift invariance of subspaces of fixed $k$ and $\left(n_{1}, n_{2}\right)$ but with an increased redundancy by a factor of four. However, the property of shift invariance is very important and does not hold true either for orthonormal waveatoms or for directional waveatoms. Ultimately, shift invariance tends to eliminate the effect of aliasing as there is no overlapping during the 'wrapping' process.

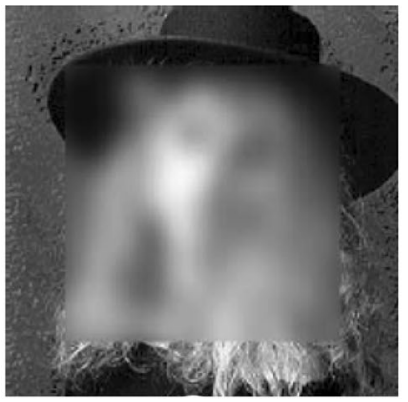

(a)

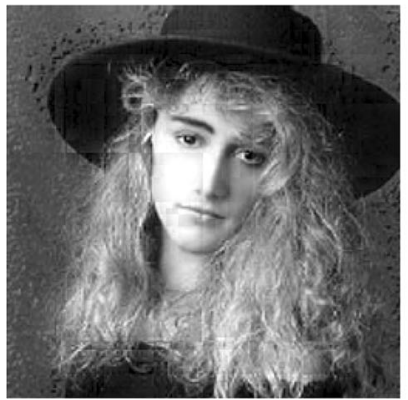

(c)

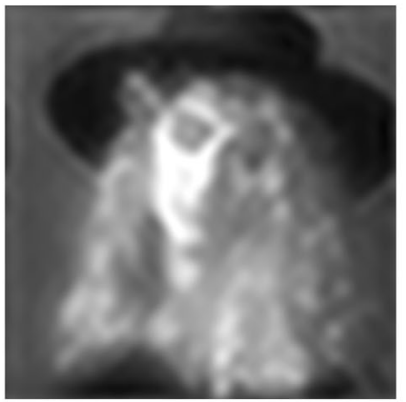

(g)

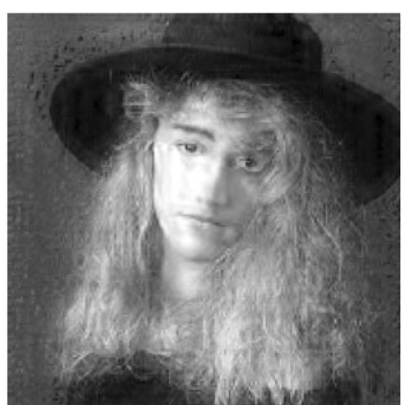

(d)

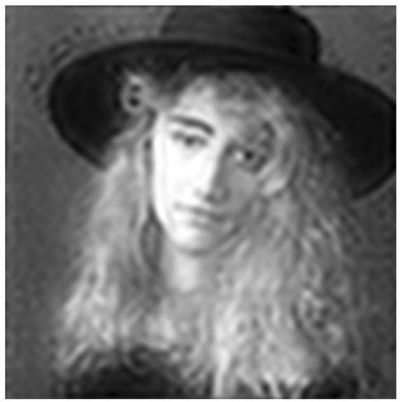

(h)

\section{Significance of visibility of an image}

Research works on human visual system have provided a mathematical model about how humans perceive this world. The application of human visual system in image processing has been explored in many research papers. Inspired from the human visual system, visibility of an image was introduced by Huang et al and later modified by Li et al [19]. Mathematically, visibility of an image $(X)$ is defined as:

$$
\operatorname{Vis}(X)=\frac{1}{P Q} \sum_{p=1}^{P} \sum_{q=1}^{Q} \frac{\left|X(p, q)-X_{\text {mean }}\right|}{X_{\text {mean }}^{c+1}}
$$

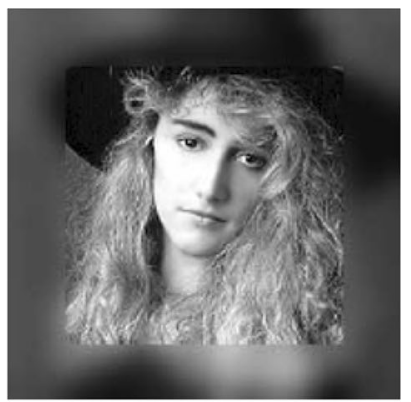

(b)

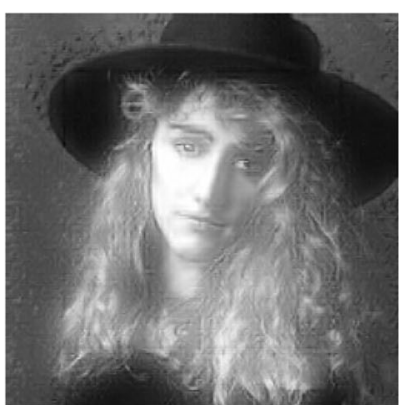

(e)

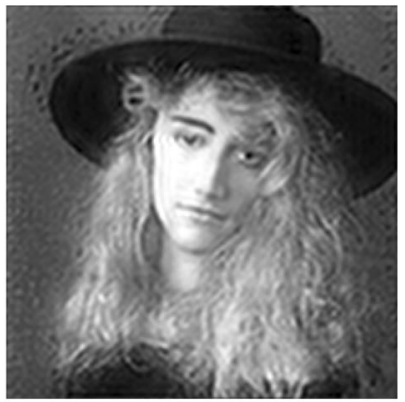

(i)

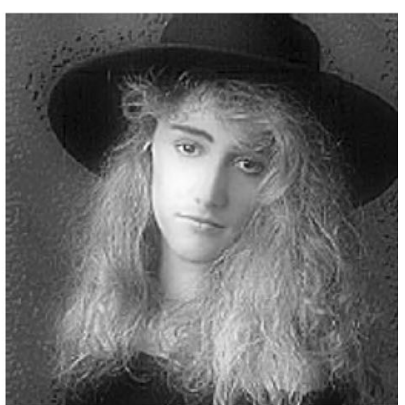

(f)

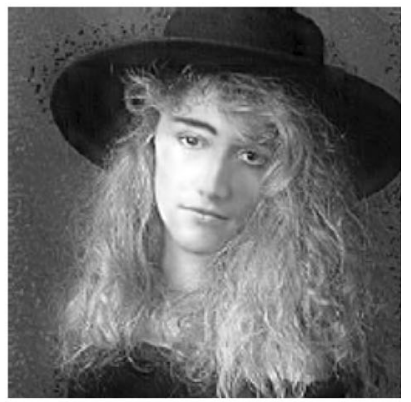

(j)

Figure 6. Fusion results for experimental image set 1 (Girl): (a) input image $(X)$, (b) input image $(Y)$; fused image using (c) DWT, (d) DCxWT, (e) DTCWT and (f) NSCT; (g) proposed algorithm (Level 1); (h) proposed algorithm (Level 2); (i) proposed algorithm (Level 3) and (j) proposed algorithm (Level 4). 
where $P$ and $Q$ are, respectively, the number of rows and columns in the image $X, c$ is a visual constant with value between 0.6 and 0.7 and $X_{\text {mean }}$ is the mean grey value of the image $X$.

Blurriness in an image commonly occurs due to the degradation of visual information. To understand the significance of visibility in the process of image fusion, the change in the values of visibility is analysed with respect to the change in blurriness in an image. For this purpose, two test images, 'Cameraman' and 'Lena', are considered. These images are first degraded by blurring using circular averaging filters of different radii (shown in figure 2) and also using motion blur with point spread function (psf) of different lengths (shown in figure 3) followed by the calculation of visibility for both types of degradation (shown in figure 4). It is observed from figures 2 and 3 that increasing the radius (in case of circular averaging filters) or length (in case of motion blur) results in increased blurriness. It is further observed from figure 4 that blurriness present in an image raises or lowers the curtain. Lower value of blurriness raises the curtain and allows clear visibility of all the details present in an image. On the other hand, higher value of blurriness lowers the curtain and reduces the visibility of details present in an image. Thus, visibility is to be considered as an important parameter of an image to be used for the fusion of multifocus images.

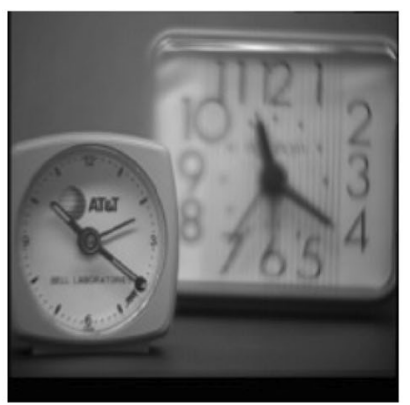

(a)

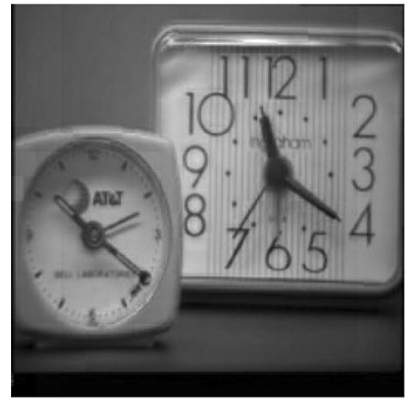

(c)

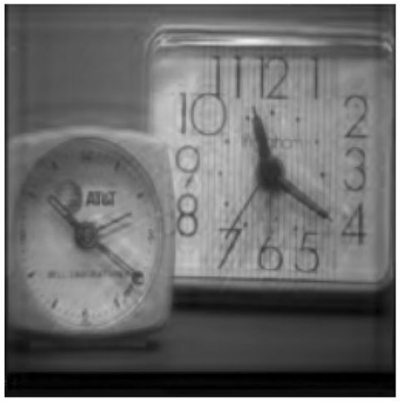

(d)

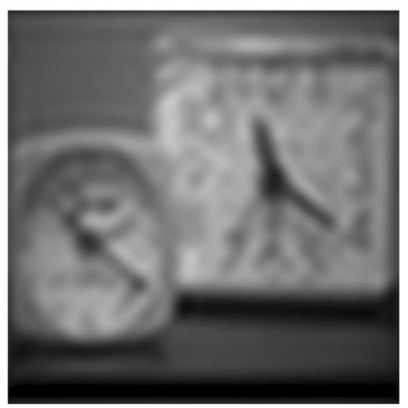

(g)

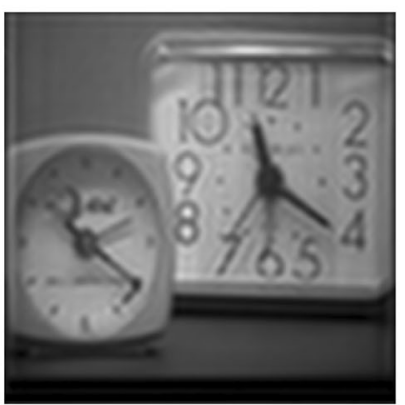

(h)

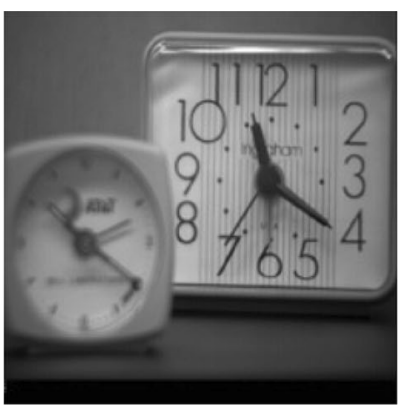

(b)

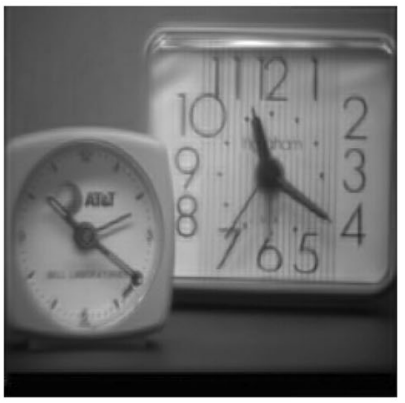

(e)

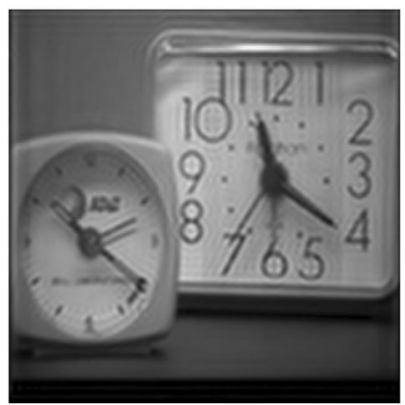

(i)

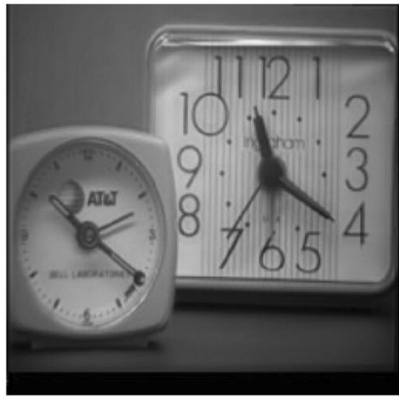

(f)

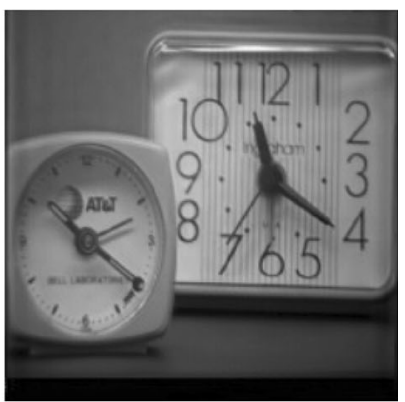

(j)

Figure 7. Fusion results for experimental image set 2 (Clocks): (a) input image $(X)$; (b) input image $(Y)$; fused image using (c) DWT, (d) DCxWT, (e) DTCWT and (f) NSCT; (g) proposed algorithm (Level 1); (h) proposed algorithm (Level 2); (i) proposed algorithm (Level 3) and (j) proposed algorithm (Level 4). 


\section{Proposed method}

Multifocus image fusion method combines partially focused input images into a single 'all-in-focus' fused image that provides an enhanced view of the same scene. Since there have been many applications such as image denoising, fingerprint identification, image compression, watermarking, etc. that explored the potential capability of waveatoms [15-18], waveatom-transform-based multifocus image fusion method has been proposed.

Once images are decomposed using waveatom transform, an appropriate fusion rule is selected to combine waveatom coefficients of input images. In the proposed work, the main aim is to perform fusion of multifocus images where the in-focus (sharper) information is to be preserved and out-of-focus (blurred) information is to be suppressed. Since visibility and blurring are inversely

proportional to each other, waveatom coefficients with maximum visibility in the neighbourhood are selected to produce the desired result.

The block diagram of the proposed method is shown in figure 5 and is detailed as follows:

1. Partially focused input images $(X$ and $Y$ ) are first decomposed at different levels using waveatom transform.

2. Calculate visibility (Eq. (7)) of each waveatom coefficient in a neighbourhood of size $3 \times 3$, centred over it.

3. Fuse waveatom coefficients of input images using the highest-visibility-based fusion rule, i.e.

$$
W_{F}(k, l)=\left\{\begin{array}{lll}
W_{X}(k, l) & \text { if } & \operatorname{Vis}\left(W_{X}\right) \geq \operatorname{Vis}\left(W_{Y}\right) \\
W_{Y}(k, l) & \text { if } & \operatorname{Vis}\left(W_{X}\right)<\operatorname{Vis}\left(W_{Y}\right)
\end{array}\right.
$$

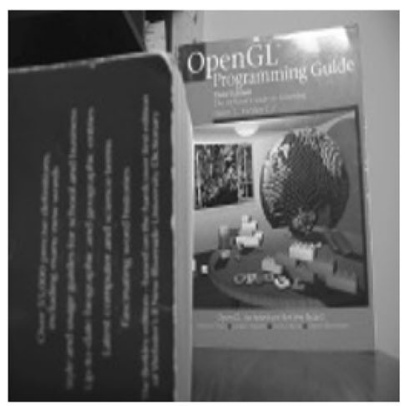

(b)

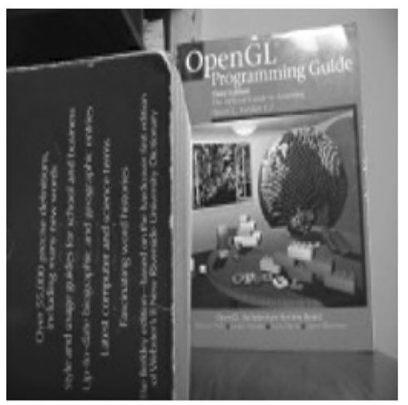

(c)

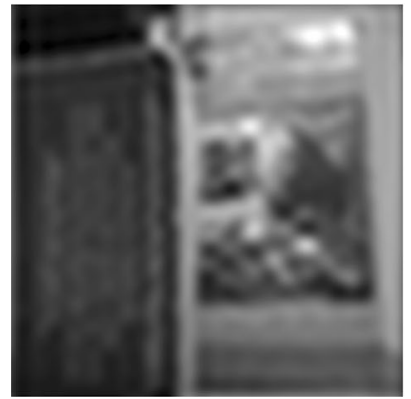

(g)

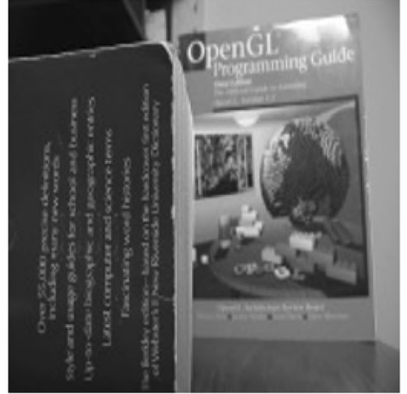

(a)

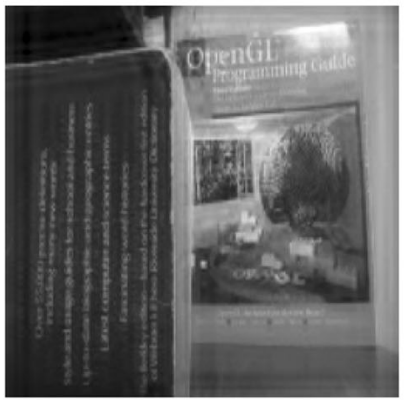

(d)

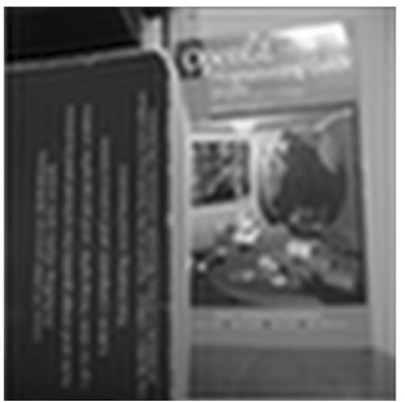

(h)

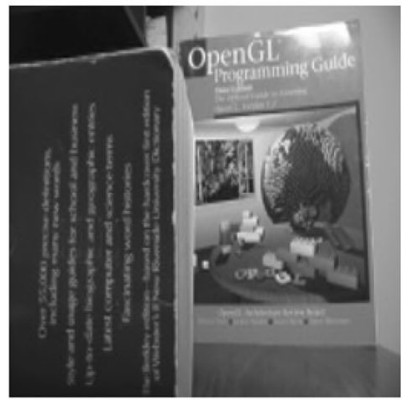

(e)

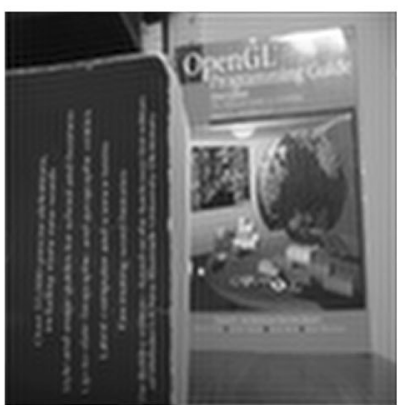

(i)

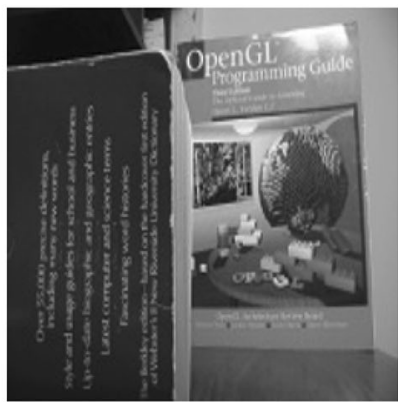

(f)

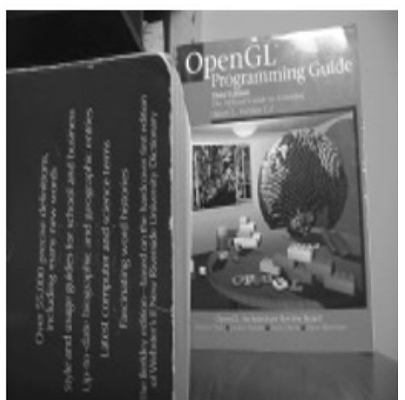

(j)

Figure 8. Fusion results for experimental image set 3 (Books): (a) input image $(X)$; (b) input image $(Y)$; fused image using (c) DWT, (d) DCxWT, (e) DTCWT and (f) NSCT; (g) proposed algorithm (Level 1), (h) proposed algorithm (Level 2), (i) proposed algorithm (Level 3) and (j) proposed algorithm (Level 4). 
where $W_{X}(k, l), W_{Y}(k, l)$ and $W_{F}(k, l)$ represent the wave-atom coefficient of input images $(X \& Y)$ and fused image $F$ at location $(k, l)$, respectively. $\operatorname{Vis}\left(W_{X}\right)$ and $\operatorname{Vis}\left(W_{Y}\right)$ represent the visibility of waveatom coefficient of input images $X$ and $Y$, respectively.

4. Perform inverse waveatom transform to produce the final 'all-in-focus' fused image $(F)$.

\section{Results and discussion}

In this paper, experiments are performed over different pairs of multifocus input images of size $256 \times 256$. The proposed method is also compared to some recent image fusion methods based on DWT [20], DTCWT [21], DCxWT [22] and NSCT [23]. Sometimes the resultant fused images obtained from different fusion methods are too similar to be distinguished by human observers, and therefore various quality measures [24, 25] such as edge strength $(E S)$, fusion loss $(F L)$, fusion artefact $(F A)$, entropy $(H)$, standard deviation $(S D)$, fusion factor $(F F)$, fusion symmetry $(F S)$, feature mutual information $(F M I)$, structural similarity index measure (SSIM) and feature similarity index measure (FSIM) are also used to objectively evaluate the performance of various methods.

1. ES measures the similarity between edges that have been transferred from input images into the fused image and is given as

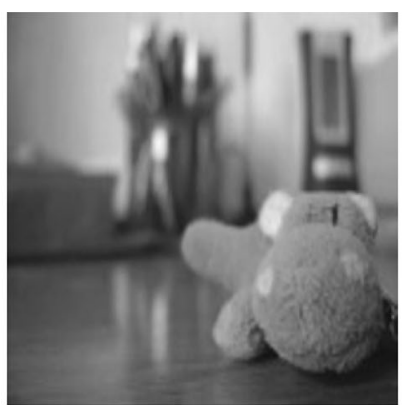

(a)

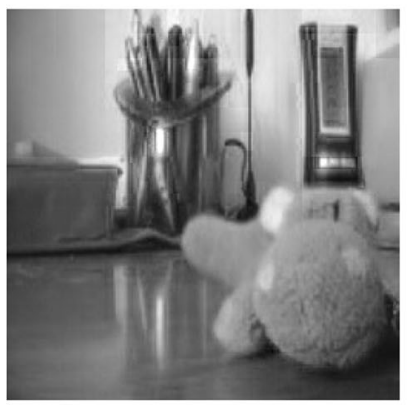

(c)

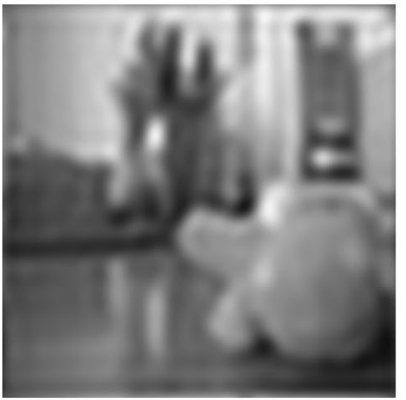

(g)

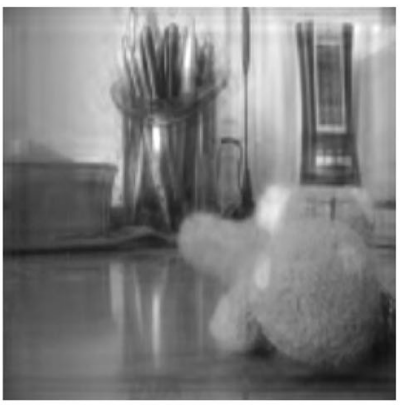

(d)

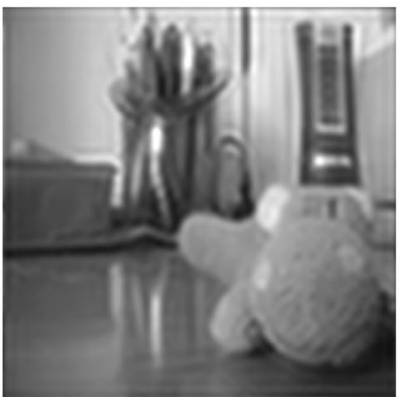

(h)

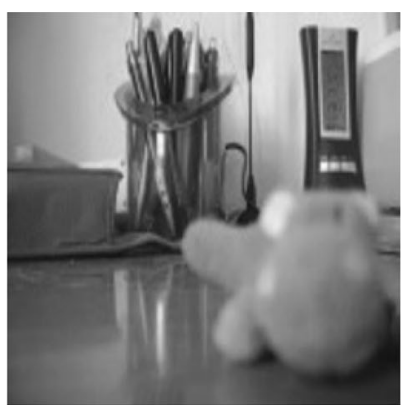

(b)

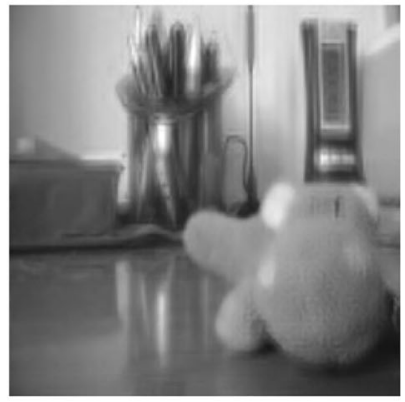

(e)

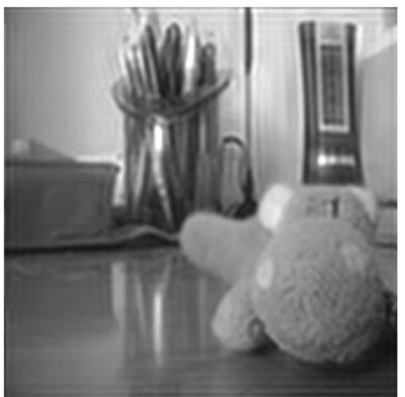

(i)

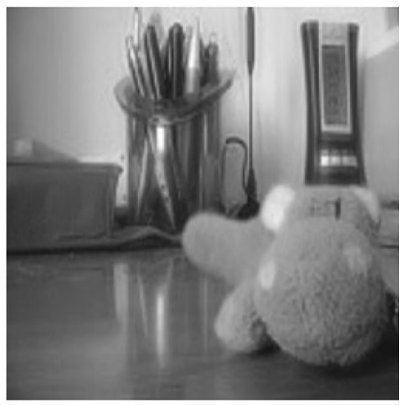

(f)

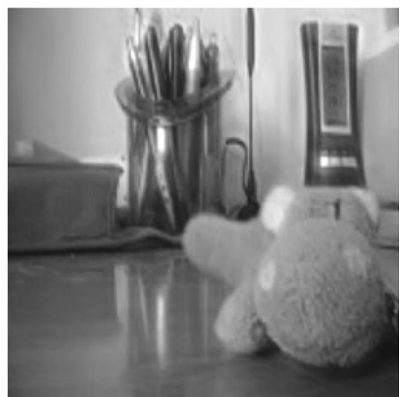

(j)

Figure 9. Fusion results for experimental image set 4 (Toy): (a) input image $(X)$, (b) input image $(Y)$; fused image using (c) DWT, (d) DCxWT, (e) DTCWT and (f) NSCT; (g) proposed algorithm (Level 1), (h) proposed algorithm (Level 2), (i) proposed algorithm (Level 3) and (j) proposed algorithm (Level 4). 


$$
\begin{aligned}
& E S= \\
& \frac{\sum_{p=1}^{P} \sum_{q=1}^{Q}\left(w_{X}(p, q) E p_{X F}(p, q)+w_{Y}(p, q) E p_{Y F}(p, q)\right)}{\sum_{p=1}^{P} \sum_{q=1}^{Q}\left(w_{X}(p, q)+w_{Y}(p, q)\right)}
\end{aligned}
$$

where $P$ and $Q$ are the number of rows and columns, respectively, in the image; $w_{X}(p, q)$ and $w_{Y}(p, q)$ are the gradient strengths of input images $X$ and $Y$, respectively. $E p_{X F}(p, q)$ and $E p_{Y F}(p, q)$ are the edge preservation values of input images $X$ and $Y$, respectively.

2. $F L$ is the amount of information that is lost during the fusion and is calculated as

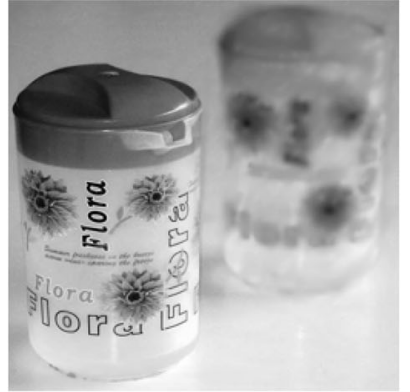

(a)

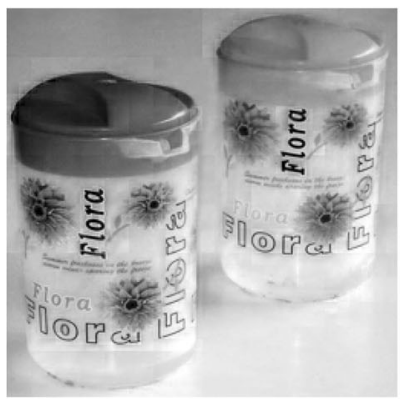

(c)

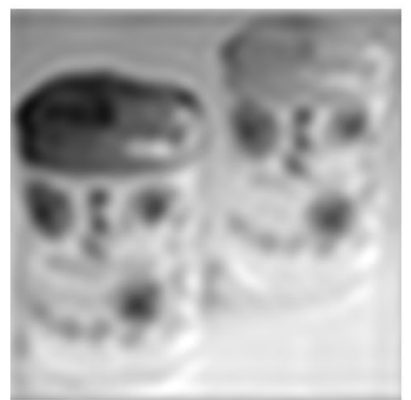

(g)

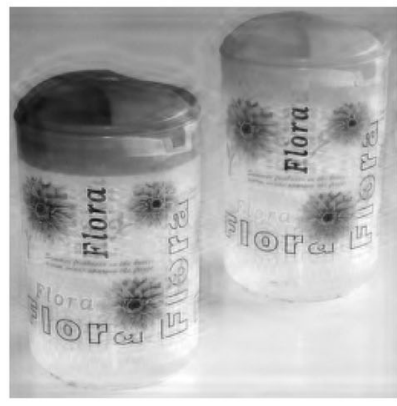

(d)

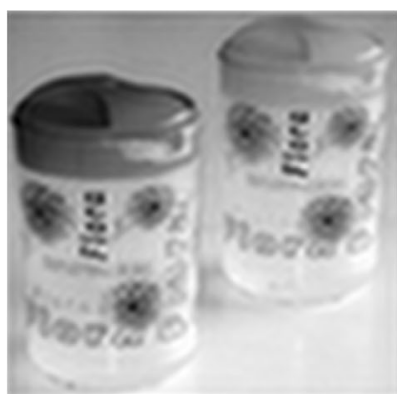

(h)

$$
F L=\frac{\sum_{p=1}^{P} \sum_{q=1}^{Q} L(p, q) x_{X Y}(p, q)}{\sum_{p=1}^{P} \sum_{q=1}^{Q}\left(w_{X}(p, q)+w_{Y}(p, q)\right)}
$$

where

$$
x_{X Y}(p, q)=\sum_{i=X, Y}\left(w_{i}(p, q)\left(1-Q_{i F}(p, q)\right)\right.
$$

and

$L(p, q)=\left\{\begin{array}{cc}1 & \text { if } s_{F}(p, q)<s_{X}(p, q) \text { or } s_{F}(p, q)<s_{Y}(p, q) \\ 0 & \text { else }\end{array}\right.$

where $s^{X}(p, q), s^{Y}(p, q)$ and $s^{F}(p, q)$ represent the $E S$ at position $(p, q)$ in $X, Y$ and $F$, respectively.

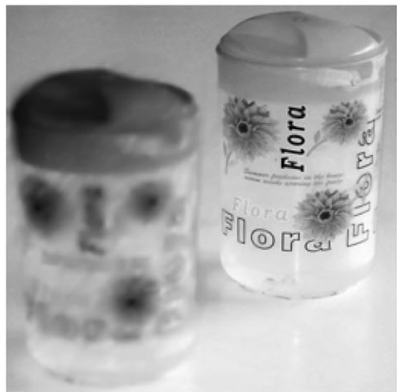

(b)

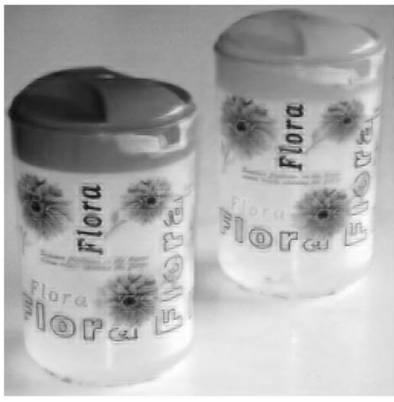

(e)

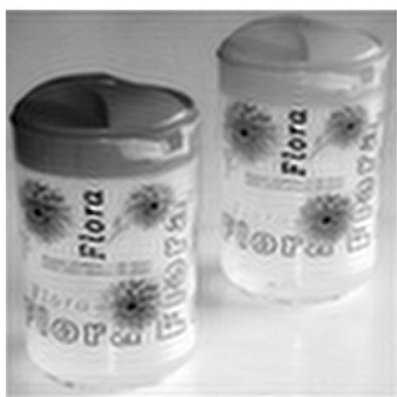

(i)

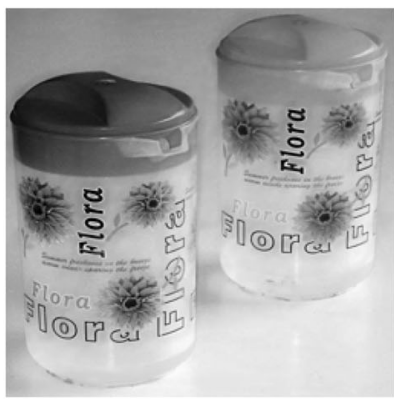

(f)

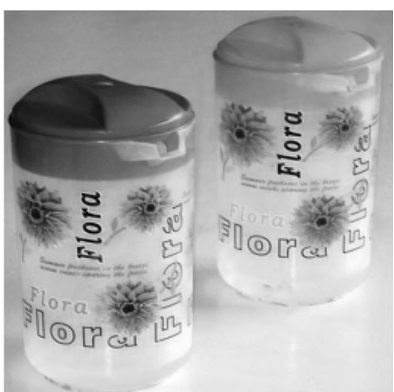

(j)

Figure 10. Fusion results for experimental image set 5 (Jugs): (a) input image $(X)$, (b) input image $(Y)$; fused image using (c) DWT, (d) DCxWT, (e) DTCWT and (f) NSCT; (g) proposed algorithm (Level 1), (h) proposed algorithm (Level 2), (i) proposed algorithm (Level 3) and (j) proposed algorithm (Level 4). 
3. FA measures the amount of noise or artefacts introduced in the fused image due to the fusion process and is given as

$$
F A=\frac{\sum_{p=1}^{P} \sum_{q=1}^{Q} A(p, q) x_{X Y}(p, q)}{\sum_{p=1}^{P} \sum_{q=1}^{Q}\left(w_{X}(p, q)+w_{Y}(p, q)\right)}
$$

where

$$
\begin{aligned}
& A(p, q)= \\
& \left\{\begin{array}{lc}
1 & \text { if } s_{F}(p, q)>s_{X}(p, q) \text { and } s_{F}(p, q)>s_{Y}(p, q) \\
0 & \text { else }
\end{array}\right.
\end{aligned}
$$

4. Entropy $(H)$ indicates the total information contained in an image and is given as

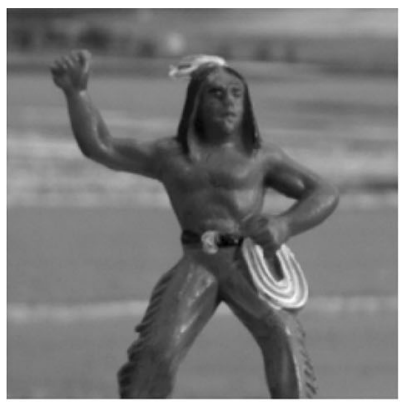

(a)

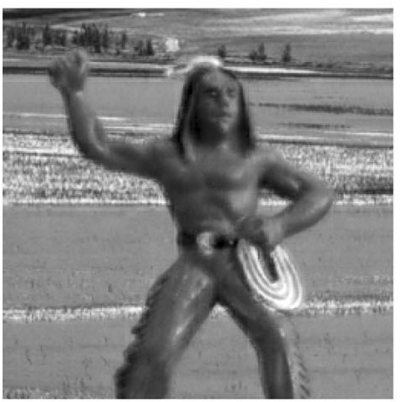

(c)

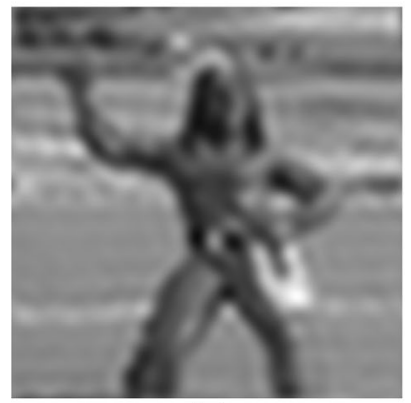

(g)

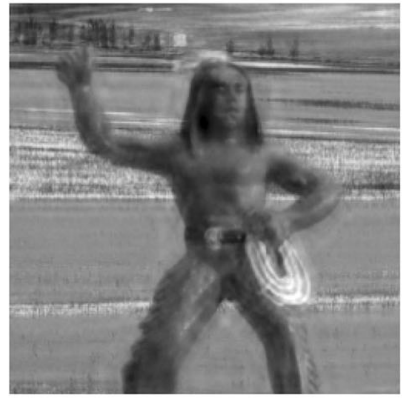

(d)

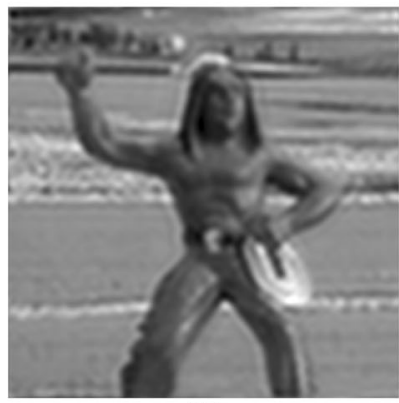

(h)

$$
H=\sum_{r=0}^{255} p_{r} \log _{2}\left(\frac{1}{p_{r}}\right)
$$

where $p_{r}$ is the probability of intensity level $r$ in an 8-bit image.

5. $S D$ reflects the spread in pixel intensity and is given as

$$
S D=\sqrt{\frac{\sum_{p=1}^{P} \sum_{q=1}^{Q}(f(p \cdot q)-\bar{F})}{P Q} .}
$$

6. FF, while fusing two images, is determined as

$$
F F=M I_{X F}+M I_{Y F} .
$$

A higher $F F$ indicates good fusion result.

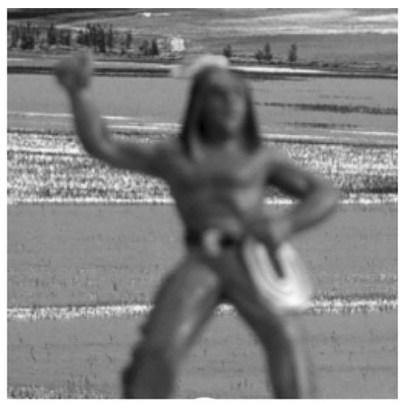

(b)

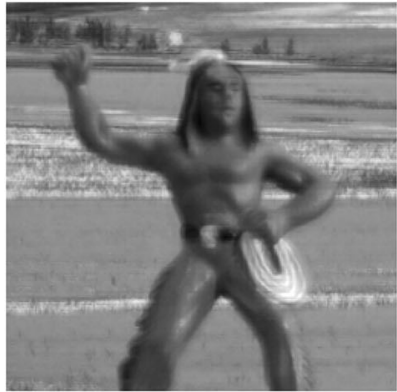

(e)

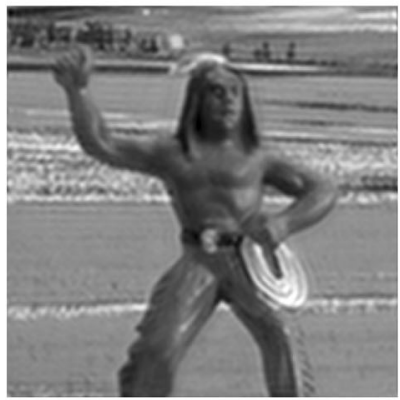

(i)

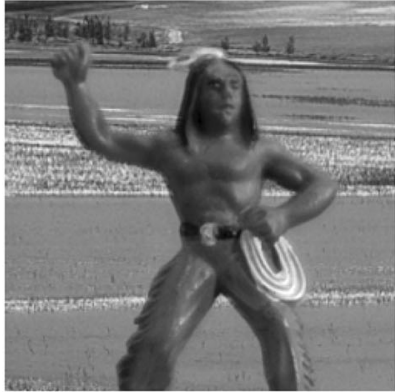

(f)

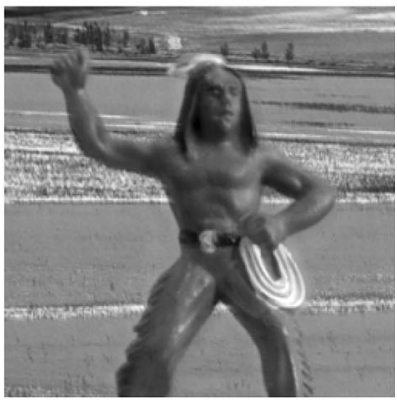

(j)

Figure 11. Fusion results for experimental image set 6 (Man): (a) input image $(X)$, (b) input image ( $Y$ ); fused image using (c) DWT, (d) DCxWT, (e) DTCWT and (f) NSCT; (g) proposed algorithm (Level 1), (h) proposed algorithm (Level 2), (i) proposed algorithm (Level 3) and (j) proposed algorithm (Level 4). 


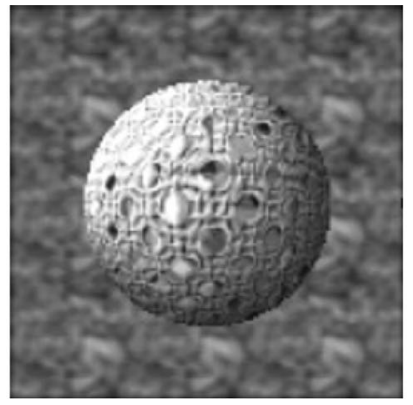

(a)

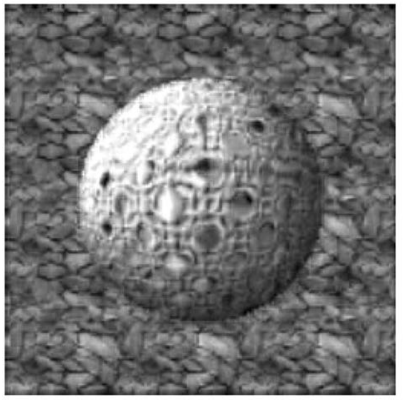

(c)

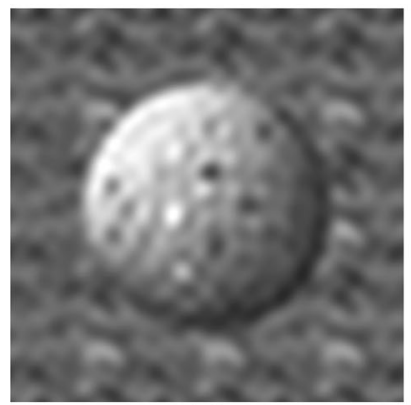

(g)

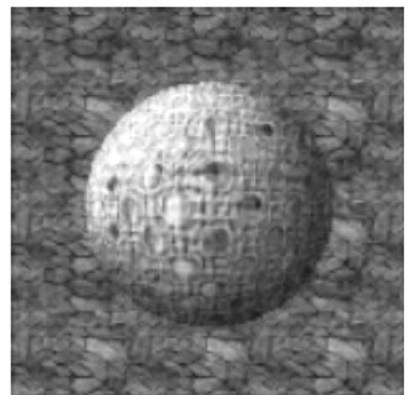

(d)

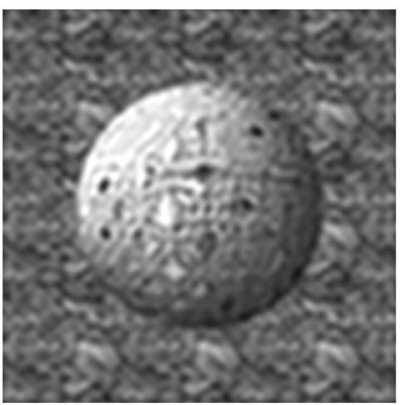

(h)

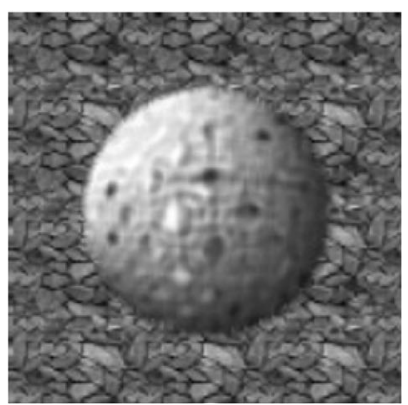

(b)

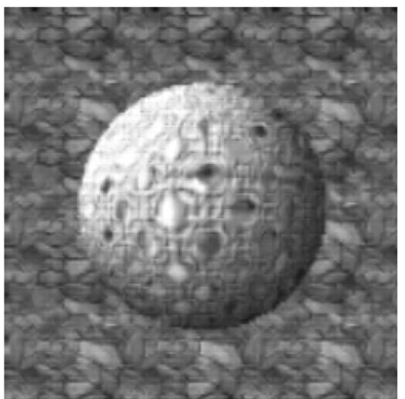

(e)

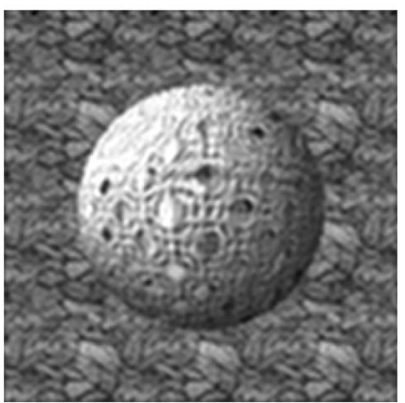

(i)

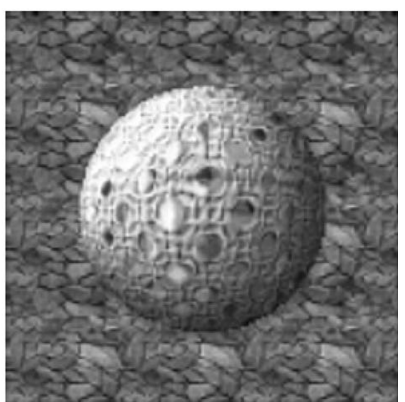

(f)

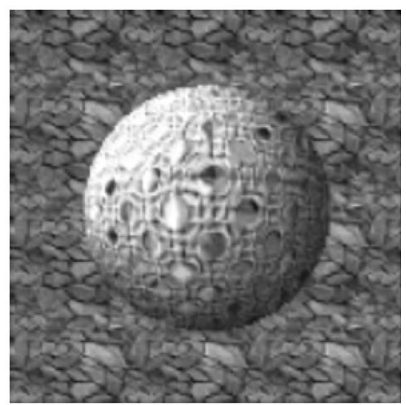

(j)

Figure 12. Fusion results for experimental image set 7 (Planet): (a) input image $(X)$, (b) input image $(Y)$; fused image using (c) DWT, (d) DCxWT, (e) DTCWT and (f) NSCT; (g) proposed algorithm (Level 1), (h) proposed algorithm (Level 2), (i) proposed algorithm (Level 3) and (j) proposed algorithm (Level 4).

7. FS signifies how much symmetric the fused image is with respect to input images and is given as

$$
F S=0.5 \frac{|R-1|}{R+1}
$$

where

$$
R=\frac{M I_{X F}}{M I_{Y F}}
$$

and $M I_{X F}$ is the mutual information between input image $X$ and the fused image $F$. Similarly, $M I_{Y F}$ is the mutual information between input image $Y$ and the fused image $F$.
8. FMI measures the quantity of feature information transferred from input images into the fused image and is defined as

$$
F M I=F_{I}(X, F)+F_{I}(Y, F)
$$

where $F_{I}(Z, F)$ is the amount of feature information transferred from image $Z$ into $F$.

9. SSIM indicates the amount of similarity between two images taking into account luminance similarity, structure similarity and contrast similarity. It is calculated as follows: 


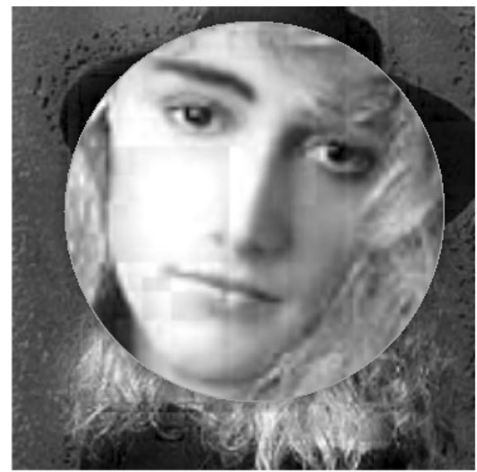

(a)

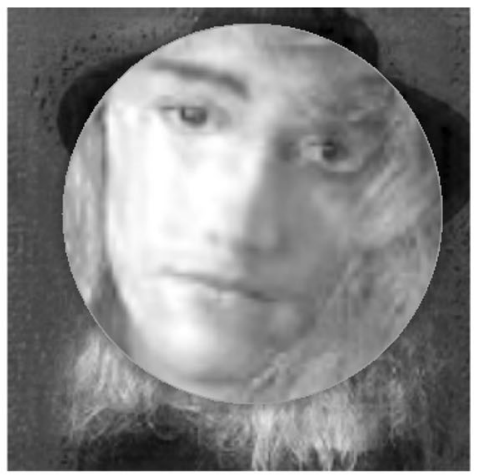

(b)

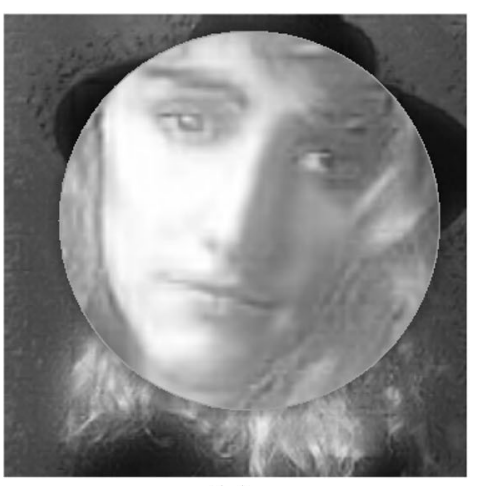

(c)

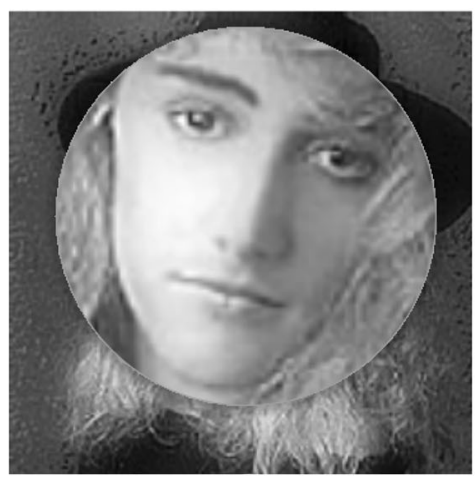

(d)

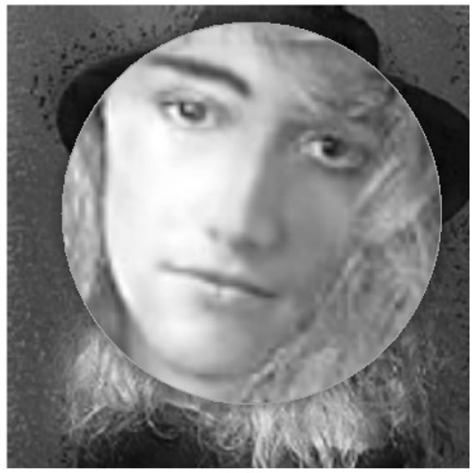

(e)

Figure 13. Zoomed results of fused images for experimental image set 1 (Girl) obtained using (a) DWT, (b) DCxWT, (c) DTCWT and (d) NSCT and (e) proposed algorithm.

$$
\begin{aligned}
\operatorname{SSIM}_{X, F}= & \left(\frac{2 m_{X} m_{F}+c_{1}}{m_{X}^{2}+m_{F}^{2}+c_{1}}\right)^{a} \times\left(\frac{\sigma_{X F}+c_{2}}{\sigma_{X} \sigma_{F}+c_{2}}\right)^{b} \\
& \times\left(\frac{2 \sigma_{X} \sigma_{F}+c_{3}}{\sigma_{X}^{2}+\sigma_{F}^{2}+c_{3}}\right)^{c}
\end{aligned}
$$

where $m_{X}$ and $m_{F}$ are the mean values of $X$ and $F$, respectively, $\sigma_{X}$ and $\sigma_{F}$ are the $S D$ of $X$ and $F$, respectively, $\sigma_{X F}$ is the covariance between $X$ and $F$ and $c_{1}, c_{2}, c_{3}$ are small constants. The first term in this equation indicates the luminance similarity, the second term indicates the structure similarity and the third term indicates the contrast similarity. The parameter $a, b$ and $c$ are responsible for adjusting the contribution of three similarities.

10. Feature similarity measure index (FSIM) is used for evaluating the similarity between images based on the use of phase congruency $(P C)$ and gradient values $(G)$ and is given as

$$
F S I M=\frac{\sum_{p=1}^{P} \sum_{q=1}^{Q} S(p, q) \max \left(P C_{X}(p, q), P C_{F}(p, q)\right.}{\sum_{p=1}^{P} \sum_{q=1}^{Q} \max \left(P C_{X}(p, q), P C_{F}(p, q)\right.}
$$

where $S(p, q)$ is the local similarity value defined in [26].
It is to be noted that larger values of $E S$, entropy $(\mathrm{H}), S D$, $F M I, F F, S S I M$ and FSIM whereas smaller values of $F L$, $F A$ and $F S$ are preferable values for evaluating the performance of fusion methods. Further, the quality measures $E S, F L$ and $F A$ should be complementary, i.e., the sum of all these should be unity [25].

\section{Results analysis}

For performance evaluation, the proposed method is experimented on different sets (set 1-set 7) of multifocus images named as 'Girl', 'Clocks', 'Books', 'Toy', 'Jugs' 'Man' and 'Planet'. Input images and the fusion results of these images using various fusion methods are shown in figures 6-12. Input images contain objects at different distances from the imaging device and so only the region that is within the 'depth-of focus' appears sharp whereas remaining of the region appears blurred. For example, figure 6a shows an image with centre region blurred in vision and 'out-of-focus' whereas the border region is sharp in vision and 'in-focus'. On the other hand, figure $6 \mathrm{~b}$ shows an image with centre region clear in vision and in-focus whereas the border region is blurred in vision and out-offocus. Figures $7 \mathrm{a}-10 \mathrm{a}$ are near-focused images where the objects near the imaging device are sharp in vision and 'infocus' whereas objects far off to the imaging device are blurred in vision and 'out-of-focus'. Figures $7 b-10 b$ are far-focused images and the situations are contrary, i.e., the 


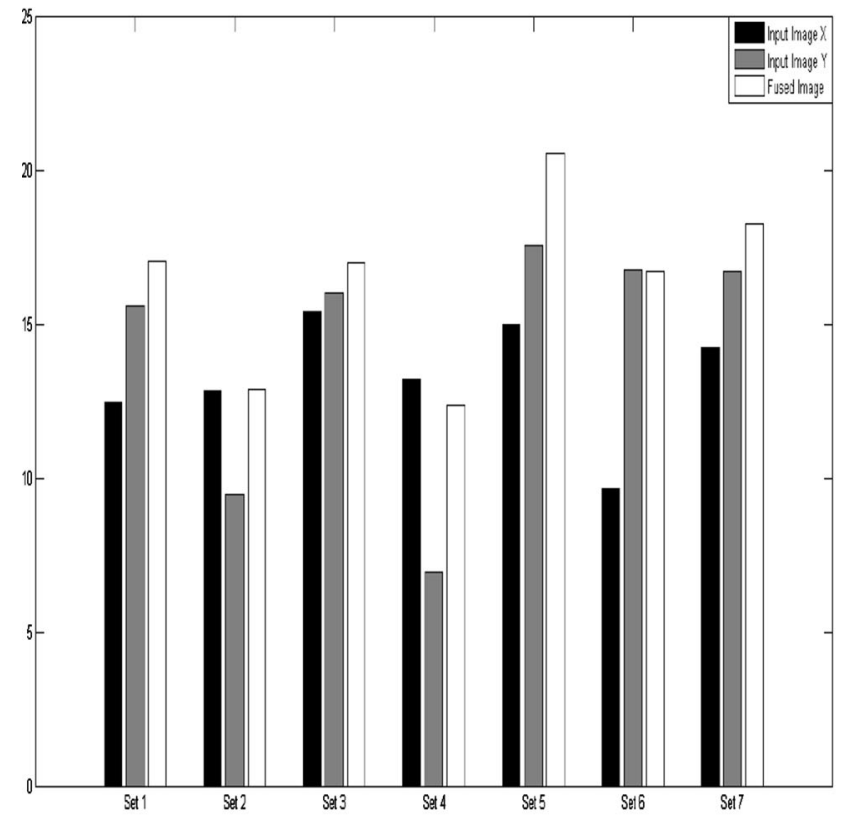

(a)

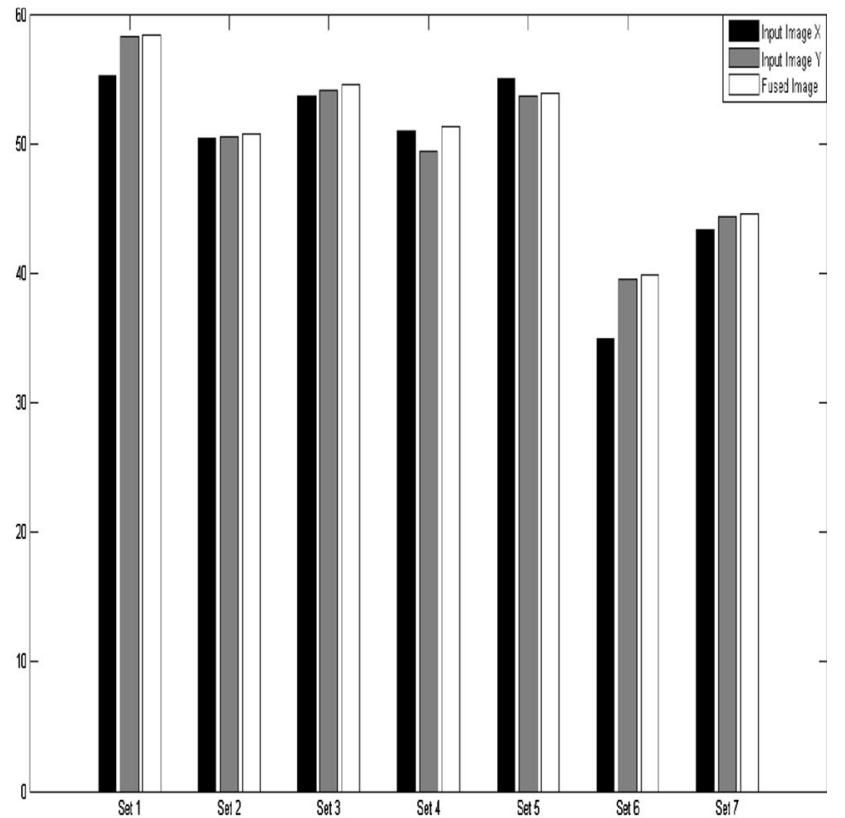

(b)

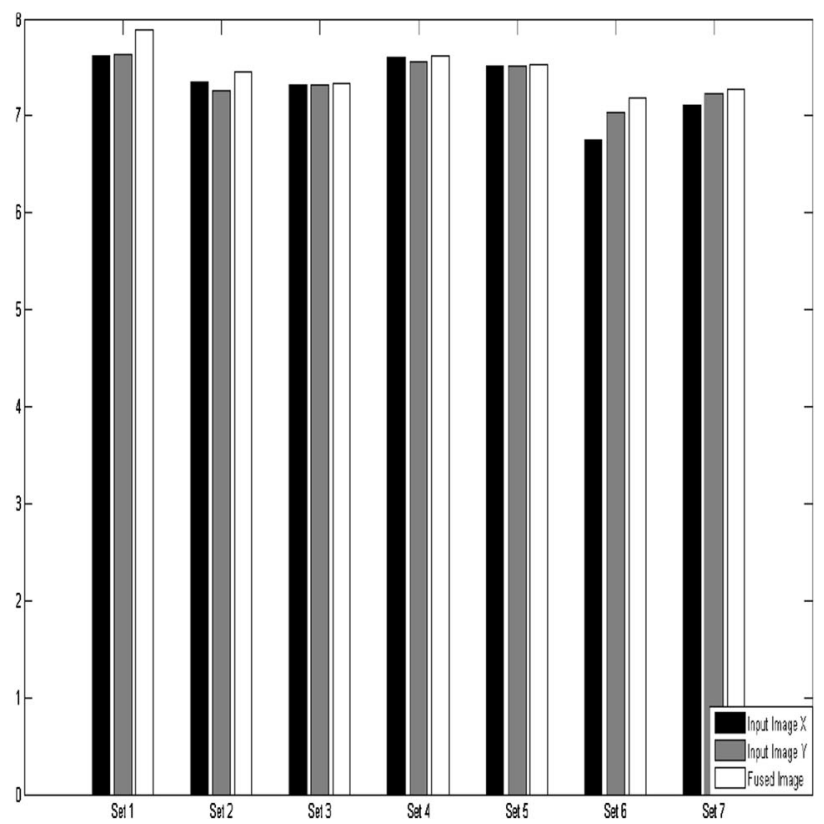

(c)

Figure 14. Objective evaluation of fused images obtained using proposed algorithm with respect to input images in terms of (a) $S F$, (b) $S D$ and (c) $H$.

objects near the imaging device are blurred in vision and 'out-of-focus' whereas objects far off to the imaging device are sharp in vision and 'in-focus'. However, figures 11a and 12a show images with foreground 'in-focus' whereas figures $11 \mathrm{~b}$ and $12 \mathrm{~b}$ show images with background 'in-focus'. The proposed fusion method has also been experimented at different scales and the results for the same are shown in figures 6-12 for different sets of multifocus images.
Figures $6 \mathrm{c}, 7 \mathrm{c}, 8 \mathrm{c}, 9 \mathrm{c}, 10 \mathrm{c}, 11 \mathrm{c}$ and $12 \mathrm{c}$ show the fused images obtained using DWT-based fusion method for different sets of multifocus images: 'Girl', 'Clocks', 'Books', 'Toy', 'Jugs' 'Man' and 'Planet', respectively. Figures 6d$12 \mathrm{~d}$ show the fusion results obtained using DCxWT-based fusion method for these sets of multifocus images. Figures $6 \mathrm{e}-12 \mathrm{e}$ show the fusion results obtained using DTCWT-based fusion method. Figures $6 \mathrm{f}-12 \mathrm{f}$ show the 
Table 1. Comparison of quality measures for fused images of set 1 (Girl).

\begin{tabular}{|c|c|c|c|c|c|c|c|c|c|c|}
\hline Methods & $E S$ & $F L$ & $F A$ & $H$ & $S D$ & $F F$ & $F S$ & $F M I$ & SSIM & FSIM \\
\hline DWT & 0.8906 & 0.0807 & 0.0387 & 7.7189 & 61.4152 & 0.8109 & 5.1349 & 0.0048 & 0.6755 & 0.7463 \\
\hline DCxWT & 0.7428 & 0.2440 & 0.0132 & 7.7066 & 58.4403 & 0.7993 & 4.7441 & 0.0059 & 0.6876 & 0.7753 \\
\hline DTCWT & 0.6695 & 0.3225 & 0.0081 & 7.6891 & 58.8619 & 0.7966 & 5.2194 & 0.0144 & 0.7176 & 0.7940 \\
\hline NSCT & 0.9109 & 0.0782 & 0.0109 & 7.6473 & 57.1193 & 0.8108 & 4.9957 & 0.0036 & 0.7134 & 0.7647 \\
\hline Proposed & 0.9167 & 0.0747 & 0.0086 & 7.8922 & 58.3947 & 0.8129 & 5.0351 & 0.0027 & 0.7199 & 0.7640 \\
\hline
\end{tabular}

Table 2. Comparison of quality measures for fused images of set 2 (Clocks).

\begin{tabular}{lcccccccccc}
\hline Methods & $E S$ & $F L$ & $F A$ & $H$ & $S D$ & $F F$ & $F S$ & $F M I$ & SSIM & $F S I M$ \\
\hline DWT & 0.8938 & 0.0862 & 0.0100 & 7.3857 & 50.5109 & 0.8803 & 6.6078 & 0.0258 & 0.9039 & 0.9134 \\
DCxWT & 0.8280 & 0.1631 & 0.0090 & 7.4544 & 49.4191 & 0.8585 & 5.6142 & $\mathbf{0 . 0 0 1 7}$ & 0.8578 & 0.8908 \\
DTCWT & 0.8262 & 0.1715 & $\mathbf{0 . 0 0 2 4}$ & 7.3740 & 50.2788 & 0.8645 & 7.1883 & 0.0110 & $\mathbf{0 . 9 2 5 8}$ & 0.9276 \\
NSCT & 0.9079 & 0.0862 & 0.0059 & 7.3548 & 50.0943 & 0.8825 & $\mathbf{7 . 2 4 7 1}$ & 0.0247 & 0.9170 & 0.9212 \\
Proposed & $\mathbf{0 . 9 1 1 2}$ & $\mathbf{0 . 0 8 3 8}$ & 0.0051 & $\mathbf{7 . 4 5 6 6}$ & $\mathbf{5 0 . 6 9 6 4}$ & $\mathbf{0 . 8 8 4 4}$ & 6.8212 & 0.0350 & 0.9077 & $\mathbf{0 . 9 2 8 2}$
\end{tabular}

Table 3. Comparison of quality measures for fused images of set 3 (Books).

\begin{tabular}{|c|c|c|c|c|c|c|c|c|c|c|}
\hline Methods & $E S$ & $F L$ & $F A$ & $H$ & $S D$ & $F F$ & $F S$ & $F M I$ & SSIM & FSIM \\
\hline DWT & 0.9644 & 0.0214 & 0.0142 & 7.3790 & 53.9517 & 0.9015 & 7.9836 & 0.0006 & 0.9722 & 0.9752 \\
\hline DCxWT & 0.9097 & 0.0848 & 0.0055 & 7.3222 & 52.1468 & 0.8709 & 5.4446 & 0.0075 & 0.8767 & 0.9035 \\
\hline DTCWT & 0.9767 & 0.0130 & 0.0003 & 7.3302 & 54.2234 & 0.9038 & 8.5187 & 0.0006 & 0.9804 & 0.9788 \\
\hline NSCT & 0.9825 & 0.0147 & 0.0028 & 7.3246 & 54.4953 & 0.9007 & 8.6007 & 0.0104 & 0.9771 & 0.9779 \\
\hline Proposed & 0.9832 & 0.0157 & 0.0010 & 7.3305 & 54.5111 & 0.9093 & 8.6030 & 0.0089 & 0.9778 & 0.9792 \\
\hline
\end{tabular}

Table 4. Comparison of quality measures for fused images of set 4 (Toy).

\begin{tabular}{lcccccccccc}
\hline Methods & $E S$ & $F L$ & $F A$ & $H$ & $S D$ & $F F$ & $F S$ & $F M I$ & SSIM & $F S I M$ \\
\hline DWT & 0.8755 & $\mathbf{0 . 0 9 7 8}$ & 0.0267 & 7.6046 & 49.6897 & 0.8949 & 6.3628 & 0.0338 & 0.8722 & 0.9034 \\
DCxWT & 0.8163 & 0.1709 & 0.0128 & 7.5979 & 49.5987 & 0.8915 & 5.1934 & $\mathbf{0 . 0 1 3 3}$ & 0.8237 & 0.8780 \\
DTCWT & 0.7592 & 0.2365 & $\mathbf{0 . 0 0 4 3}$ & 7.5887 & 50.3636 & 0.8809 & $\mathbf{7 . 0 0 6 4}$ & 0.0462 & $\mathbf{0 . 9 0 0 1}$ & 0.9111 \\
NSCT & 0.8845 & 0.1094 & 0.0060 & 7.5866 & 50.5620 & 0.9012 & 6.8516 & 0.0161 & 0.8940 & 0.9105 \\
Proposed & $\mathbf{0 . 8 8 5 2}$ & 0.1057 & 0.0091 & $\mathbf{7 . 6 1 1 5}$ & $\mathbf{5 1 . 2 5 6 3}$ & $\mathbf{0 . 9 0 1 9}$ & 6.4721 & 0.0427 & 0.8819 & $\mathbf{0 . 9 1 1 9}$ \\
\hline
\end{tabular}

Table 5. Comparison of quality measures for fused images of set 5 (Mugs).

\begin{tabular}{lcccccccccc}
\hline Methods & $E S$ & $F L$ & $F A$ & $H$ & $S D$ & $F F$ & $F S$ & $F M I$ & SSIM & $F S I M$ \\
\hline DWT & 0.8885 & 0.0887 & 0.0227 & 7.5180 & 52.1480 & 0.8871 & 6.0200 & 0.0100 & 0.8593 & 0.8902 \\
DCXWT & 0.8153 & 0.1702 & 0.0144 & 7.4653 & 52.0105 & 0.8715 & 4.8786 & 0.0068 & 0.8245 & 0.8761 \\
DTCWT & 0.8074 & 0.1840 & 0.0086 & 7.4674 & 52.8297 & 0.8720 & 6.7687 & $\mathbf{0 . 0 0 2 6}$ & 0.8800 & 0.9014 \\
NSCT & 0.9123 & 0.0770 & 0.0099 & 7.4954 & 53.7047 & $\mathbf{0 . 8 9 0 1}$ & 6.4391 & 0.0064 & 0.8807 & $\mathbf{0 . 9 0 1 7}$ \\
Proposed & $\mathbf{0 . 9 1 2 9}$ & $\mathbf{0 . 0 8 0 0}$ & $\mathbf{0 . 0 0 7 1}$ & $\mathbf{7 . 5 2 2 5}$ & $\mathbf{5 3 . 8 4 2 0}$ & 0.8876 & $\mathbf{6 . 9 0 9 7}$ & 0.0069 & $\mathbf{0 . 8 8 1 3}$ & 0.8989 \\
\hline
\end{tabular}


Table 6. Comparison of quality measures for fused images of set 6 (Man).

\begin{tabular}{|c|c|c|c|c|c|c|c|c|c|c|}
\hline Methods & $E S$ & $F L$ & $F A$ & $H$ & $S D$ & $F F$ & $F S$ & $F M I$ & SSIM & FSIM \\
\hline DWT & 0.8621 & 0.0886 & 0.0504 & 7.0946 & 37.8628 & 0.8465 & 5.0111 & 0.1098 & 0.8046 & 0.8417 \\
\hline DCxWT & 0.8288 & 0.1611 & 0.0100 & 6.9731 & 37.9127 & 0.8342 & 3.9899 & 0.0627 & 0.7739 & 0.8395 \\
\hline DTCWT & 0.7609 & 0.2327 & 0.0064 & 6.9674 & 38.5227 & 0.8214 & 4.8099 & 0.0699 & 0.8257 & 0.8474 \\
\hline NSCT & 0.9168 & 0.0744 & 0.0088 & 6.9267 & 38.3418 & 0.8471 & 5.0383 & 0.0804 & 0.8199 & 0.8486 \\
\hline Proposed & 0.9174 & 0.0751 & 0.0075 & 7.1917 & 39.8864 & 0.8506 & 5.0407 & 0.1199 & 0.8278 & 0.8495 \\
\hline
\end{tabular}

Table 7. Comparison of quality measures for fused images of set 7 (Planet).

\begin{tabular}{lcccccccccc}
\hline Methods & $E S$ & $F L$ & $F A$ & $H$ & $S D$ & $F F$ & $F S$ & FMI & SSIM & $F S I M$ \\
\hline DWT & 0.8422 & 0.1104 & 0.0474 & 7.1864 & 43.3252 & 0.8314 & 3.9579 & 0.0443 & 0.7534 & 0.8588 \\
DCxWT & 0.7505 & 0.2374 & 0.0122 & 7.1152 & 41.7780 & 0.8316 & 3.4073 & 0.0160 & 0.7282 & 0.8482 \\
DTCWT & 0.7625 & 0.2322 & $\mathbf{0 . 0 0 5 3}$ & 7.1636 & 43.0407 & 0.8287 & 4.0672 & $\mathbf{0 . 0 1 0 9}$ & $\mathbf{0 . 7 9 7 4}$ & 0.8642 \\
NSCT & 0.8873 & 0.1016 & 0.0111 & 7.2627 & 44.4205 & 0.8346 & $\mathbf{4 . 2 8 0 1}$ & 0.0652 & 0.7736 & 0.8684 \\
Proposed & $\mathbf{0 . 8 8 7 8}$ & $\mathbf{0 . 1 0 2 0}$ & 0.0103 & $\mathbf{7 . 2 7 1 4}$ & $\mathbf{4 4 . 5 7 7 1}$ & $\mathbf{0 . 8 3 7 5}$ & 4.1721 & 0.0595 & 0.7737 & $\mathbf{0 . 8 6 8 8}$
\end{tabular}

fusion results obtained using NSCT-based fusion method and finally figures $6 \mathrm{~g}-\mathrm{j}$ to $12 \mathrm{~g}-\mathrm{j}$ show the fusion results obtained using the proposed fusion method at different scales. Results obtained from the proposed method show that better quality of fused image is obtained at higher scales. It has also been experimentally verified that there is negligible improvement in the quality of fused image but at the cost of high time complexity after scale 4 and therefore results of the proposed method at scale 4 has been considered in the present study. Figure 13 shows the zoomed results of fused images obtained using different fusion methods for experimental image set 1 . From the zoomed results shown in figure 13, it is observed that DWT-based fusion method produces significant blocking artefacts; DCxWT and DTCWT result in poor visual quality of fusion results. The fused image produced by NSCT-based fusion method looks good but on close observation it is observed that NSCT-based fusion method is not able to show prominent features and also suffers from contrast reduction; the same can be observed from the comparison of hair, cheek and eyebrow of the fused image obtained using NSCT-based fusion method vis-a-vis input images. However, the fused image obtained using the proposed method successfully fuses the partially focused multiple images into a visually better fused image with all prominent features and proper contrast.

For all the sets of multifocus images, the objective evaluation (using spatial frequency, $S D$ and entropy) of fused images obtained using the proposed fusion method with respect to input images is shown in figure 14. The highest value of SF for most of the sets (shown in figure 14a) indicates that the fused images obtained using the proposed method are clearer than the respective input images. Similarly the higher values of $S D$ (shown in figure 14b) obtained using proposed method reveal better contrast of fused images than those of the input images. Further, higher values of entropy (shown in figure 14c) for the fused images indicate that the fusion results obtained using the proposed fusion method contain more information than the input images. Tables 1, 2, 3, 4, 5, 6 and 7 show the objective evaluation of various methods for different sets of multifocus images. Before analysing these objective results, it should be remembered that high values of $E S, H, S D$, $F M I, F F$, SSIM and FSIM and low values of FL, FA and FS indicate a better quality of fused image. For each set of input images, the best value of each objective measure is indicated in bold. It is observed from these tables that most of the objective measures for the proposed method have attained their desirable values for all the sets of multifocus images.

Thus, from the comparisons of various results, it is concluded that the proposed method performs well and exhibits excellent fusion capability and thereby produces a better quality of 'all-in-one' focused fused image.

\section{Conclusion}

Multifocus image fusion method combines partially focused input images into a single 'all-in-focus' fused image, providing an enhanced view of the same scene. Thus, waveatom-transform-based algorithm for fusion of multifocus images has been proposed. Since visibility is inversely proportional to the blurriness present in an image, the waveatom coefficients with higher visibility corresponding to sharper image intensities are used to perform the process of image fusion. The performance of the proposed method is evaluated visually as well as in terms of various objective measures. Results prove that the proposed method effectively fuses multifocus input images into a 
single well-focused fused image and performs better than existing fusion methods.

\section{References}

[1] Stathaki T 2008 Image fusion: algorithms and applications. Academic Press, Cambridge

[2] Li S, Kwok J T and Wang Y 2002 Multifocus image fusion using artificial neural networks. Pattern Recognit. Lett. 23: 985-997

[3] Agrawal D and Singhai J 2010 Multifocus image fusion using modified pulse coupled neural network for improved image quality. IET Image Process. 4: 443-451

[4] Manchanda M and Sharma R 2016 A novel method of multimodal medical image fusion using fuzzy transform. $J$. Vis. Commun. Image Represent. 40: 197-217

[5] Yang B, Jing Z L and Zhao H T 2010 Review of pixel-level image fusion. J. Shanghai Jiaotong Univ. (Sci.) 15: 6-12

[6] Li S and Yang B 2008 Multifocus image fusion using region segmentation and spatial frequency. Image Vis. Comput. 26: 971-979

[7] Wan T, Zhu C and Qin Z 2013 Multifocus image fusion based on robust principal component analysis. Pattern Recognit. Lett. 34: 1001-1008

[8] Wang W and Chang F 2011 A multi-focus image fusion method based on Laplacian pyramid. J. Comput. 6: 2559-2566

[9] Rahman S M M, Ahmad M O and Swamy M N S 2010 Contrast-based fusion of noisy images using discrete wavelet transform. IET Image Process. 4: 374-384

[10] Manchanda M and Sharma R 2018 An improved multimodal medical image fusion algorithm based on fuzzy transform. $J$. Vis. Commun. Image Represent. 51: 76-94

[11] Ali F E et al 2008 Curvelet fusion of MR and CT images. Progress Electromagn. Res. 3: 215-224

[12] Wang $\mathbf{J}$ et al 2013 Image fusion with nonsubsampled contourlet transform and sparse representation. J. Electron. Imaging 22: 043019(1)-043019(15)
[13] Wang L, Li B and Tian L 2013 A novel multi-modal medical image fusion method based on shift-invariant shearlet transform. Imaging Sci. J. 61: 529-540

[14] Demanet L and Ying L 2007 Wave atoms and sparsity of oscillatory patterns. Appl. Comput. Harmon. Anal. 23: 368-387

[15] Boutella L and Serir A 2013 Fingerprint orientation map based on wave atoms transform. J. Image Gr. 1: 129-133

[16] Mohammed A A et al 2010 An efficient fingerprint image compression technique based on wave atoms decomposition and multistage vector quantization. Integr. Comput. Aided Eng. 17: $29-40$

[17] Haddad, Z. et al 2013 Wave atoms based compression method for fingerprint images. Pattern Recognit. 46: 2450-2464

[18] Leung H Y and Cheng L M 2011 Robust watermarking scheme using wave atoms. EURASIP J. Adv. Signal Process. 1: 184817

[19] Li M, Cai W and Tan Z 2006 A region-based multi-sensor image fusion scheme using pulse-coupled neural network. Pattern Recognit. Lett. 27: 1948-1956

[20] Shu-Long Z 2002 Image fusion using wavelet transform. Int. Arch. Photogramm. Remote Sens. Spat. Inf. Sci. 34: 552-556

[21] Hill P R, Canagarajah C N and Bull D R 2002 Image fusion using complex wavelets. In: Proceedings of $B M V C$, vol. 1 , pp. $1-10$

[22] Singh R and Khare A 2014 Fusion of multimodal medical images using Daubechies complex wavelet transform-a multiresolution approach. Inf. Fus. 19: 49-60

[23] Zhang Q and Guo B L 2009 Multifocus image fusion using the nonsubsampled contourlet transform. Signal Process. 89: 1334-1346

[24] Kotwal K and Chaudhuri S 2013 A novel approach to quantitative evaluation of hyperspectral image fusion techniques. Inf. Fus. 14: 5-18

[25] Kumar B S 2013 Multifocus and multispectral image fusion based on pixel significance using discrete cosine harmonic wavelet transform. Signal Image Video Process. 7: 1125-1143

[26] Wang Z et al 2004 Image quality assessment: from error visibility to structural similarity. IEEE Trans. Image Process. 13: $600-612$ 